\title{
Anisotropic, adaptive finite elements for the computation of a solutal dendrite
}

\author{
E. BURMAN ${ }^{\dagger}$ AND M. PICASSO \\ Institut de Mathématiques, École Polytechnique Fédérale de Lausanne, \\ 1015 Lausanne, Switzerland
}

[Received 20 June 2002 and in revised form 31 October 2002]

\begin{abstract}
We compute solutions of solutal phase-field models for dendritic growth of an isothermal binary alloy using anisotropic mesh refinement techniques. The adaptive strategy is based on anisotropic a posteriori estimators using a superconvergent recovery technique in the form of the ZienkiewiczZhu error estimator. The phase-field model contains an anisotropic strongly nonlinear second order operator modelling the dendritic branches; this strong nonlinearity is included in the a posteriori error estimators by using a monotonicity result. The monotonicity holds for phase-field anisotropy below a certain threshold value beyond which there are no known well-posedness results. We present computational results for both regimes showing the performance of the proposed method.
\end{abstract}

\section{Introduction}

In this paper we consider the problem of computing solutions to a phase-field model for an isothermal binary alloy. The model consists of a parabolic system of nonlinear equations modelling the phase-field, $\phi$, which is an order parameter taking the value 1 in the solid phase and 0 in the liquid phase, making the transition in a continuous fashion in the thin solid-liquid interface, known as the "mushy zone" (see Fig. 1.1) and the concentration of the binary alloy $c$, also taking values between zero and one depending on the mass fraction of the alloy constituants.

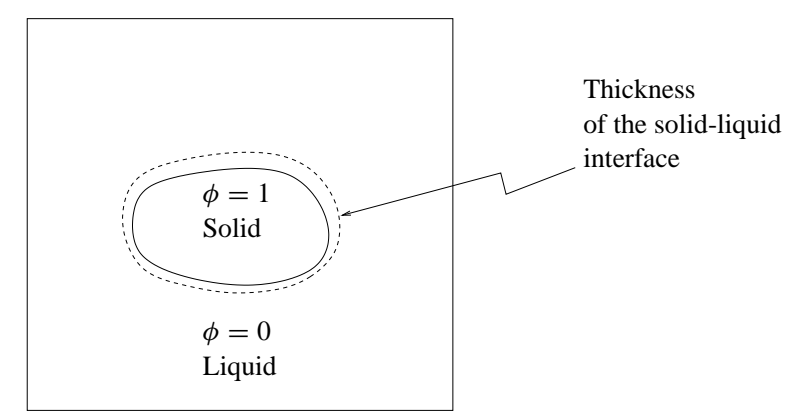

FIG. 1.1. The phase-field $\phi$.

A general mathematical formulation of this solidification problem, including the models of [23] and [22], is the following. Given a bounded domain $\Omega$ of $\mathbb{R}^{2}$ with outer unit normal $\mathbf{n}, c_{0}, \phi_{0} \in$

\footnotetext{
${ }^{\dagger}$ Corresponding author, supported by the Swiss National Foundation. Email: erik.burman@epfl.ch
}

Email: marco.picasso@epfl.ch 
$H^{1}(\Omega)$ and a time interval $(0, T)$ we consider the problem of finding $\phi, c: \Omega \times(0, T) \rightarrow \mathbb{R}$ such that

$$
\begin{array}{ll}
\left.\alpha \frac{\partial \phi}{\partial t}-\operatorname{div}(A(\nabla \phi) \nabla \phi)\right)-S(c, \phi)=0 & \text { in } \Omega \times(0, T), \\
\frac{\partial c}{\partial t}-\operatorname{div}\left(D_{1}(\phi) \nabla c+D_{2}(c, \phi) \nabla \phi\right)=0 & \text { in } \Omega \times(0, T), \\
A(\nabla \phi) \nabla \phi \cdot \mathbf{n}=0 & \text { on } \partial \Omega \times(0, T), \\
\left(D_{1}(\phi) \nabla c+D_{2}(c, \phi) \nabla \phi\right) \cdot \mathbf{n}=0 & \text { on } \partial \Omega \times(0, T), \\
\phi(0)=\phi_{0}, \quad c(0)=c_{0} & \text { in } \Omega .
\end{array}
$$

Here $\alpha \in \mathbb{R}$ is a positive parameter and $A(\cdot)$ is the matrix defined for $\xi \in \mathbb{R}^{2} \backslash 0$ by

$$
A(\xi)=\left(\begin{array}{cc}
a^{2}(\theta(\xi)) & -a(\theta(\xi)) a^{\prime}(\theta(\xi)) \\
a(\theta(\xi)) a^{\prime}(\theta(\xi)) & a^{2}(\theta(\xi))
\end{array}\right)
$$

where $\theta(\xi)$ denotes the angle between the vector $\xi$ and the first component of the orthonormal cartesian basis $\left(e_{1}, e_{2}\right)$, that is,

$$
\cos \theta(\xi)=\frac{\xi \cdot e_{1}}{\|\xi\|},
$$

and $a$ is the real-valued function defined by

$$
a(\theta)=1+\bar{a} \cos (\kappa \theta),
$$

with $\bar{a} \geqslant 0$ (the anisotropy parameter) and $\kappa$ a positive integer corresponding to the number of dendritic branching directions.

In [20] it is shown that (1.1)- -1.5 ) has a unique weak solution, in the isotropic case $\bar{a}=0$ (thus $A(\cdot)=I)$. Furthermore in this case a priori error estimates, a posteriori and adaptive finite elements are available in [16, 17]. Existence of a weak solution in the anisotropic case $\bar{a} \neq 0$ has been proved in [8]. More precisely, if $S, D_{1}$ and $D_{2}$ are bounded Lipschitz functions satisfying

$$
\begin{array}{ll}
0<D_{s} \leqslant D_{1}(\phi) \leqslant D_{l} & \forall \phi \in \mathbb{R}, \\
S(c, 0)=S(c, 1)=0 & \forall c \in \mathbb{R}, \\
D_{2}(0, \phi)=D_{2}(1, \phi)=0 & \forall \phi \in \mathbb{R},
\end{array}
$$

and if $\bar{a} \leqslant 1 /\left(\kappa^{2}-1\right)$, then problem 1.1$\left.]-1.5\right)$ has a weak solution

$$
\phi, c \in L^{2}\left(0, T ; H^{1}(\Omega)\right) \cap H^{1}\left(0, T ;\left(H^{1}(\Omega)\right)^{\prime}\right) .
$$

Moreover, if the functions $S$ and $D_{2}$ are extended by zero outside the interval $(0,1)$, then a maximum principle holds for $\phi$ and $c$. In [7] an a priori error estimate in the $L^{2}\left(0, T ; H^{1}(\Omega)\right)$ norm is proved under the same condition on $\bar{a}$ as above and assuming the existence of a sufficiently regular solution. Note that the Lipschitz constant for $S$ is proportional to the square of one over the interface thickness; for a typical example of the form of $S$ we refer to the numerical section.

Our goal is to perform anisotropic, adaptive finite element computations for solving efficiently (1.1)- $\sqrt{1.5}$ in the general case when $\bar{a} \neq 0$. Anisotropic error estimates based on the anisotropic interpolation estimates of [14,13] are derived, using techniques similar to those presented in [18, 
19. Since 1.1 is strongly nonlinear, the difficulty is to relate the true $H^{1}$ error to the equation residual.

An outline of the paper is as follows: in Section 2 we recall some results on anisotropic interpolation, in Section 3 we discretize the problem in space and derive anisotropic a posteriori error estimates. In Sections 4 and 5 we propose a time discretization and an adaptive algorithm based on a simplified anisotropic error indicator. The algorithm is then tested on some problems with varying degree of physical anisotropy $\bar{a}$ in Section 6

\section{Anisotropic interpolation estimates}

For any $0<h<1$, let $\mathcal{T}_{h}$ be a conforming triangulation of $\bar{\Omega}$ into triangles $K$ with diameter $h_{K}$ less than $h$. Let $V_{h}$ be the usual finite element space of continuous, piecewise linear functions on the triangles of $\mathcal{T}_{h}$.

For any triangle $K$ of the mesh, let $T_{K}: \hat{K} \rightarrow K$ be the affine transformation which maps the reference triangle $\hat{K}$ into $K$. Let $M_{K}$ be the Jacobian of $T_{K}$, that is,

$$
\mathbf{x}=T_{K}(\hat{\mathbf{x}})=M_{K} \hat{\mathbf{x}}+\mathbf{t}_{K} .
$$

Since $M_{K}$ is invertible, it admits a singular value decomposition $M_{K}=R_{K}^{T} \Lambda_{K} P_{K}$, where $R_{K}$ and $P_{K}$ are orthogonal and where $\Lambda_{K}$ is diagonal with positive entries. In the following we set

$$
\Lambda_{K}=\left(\begin{array}{cc}
\lambda_{1, K} & 0 \\
0 & \lambda_{2, K}
\end{array}\right) \quad \text { and } \quad R_{K}=\left(\begin{array}{l}
\mathbf{r}_{1, K}^{T} \\
\mathbf{r}_{2, K}^{T}
\end{array}\right),
$$

with the choice $\lambda_{1, K} \geqslant \lambda_{2, K}$. A simple example of such a transformation is $x_{1}=H \hat{x}_{1}, x_{2}=h \hat{x}_{2}$, with $H \geqslant h$, thus

$$
M_{K}=\left(\begin{array}{cc}
H & 0 \\
0 & h
\end{array}\right), \quad \lambda_{1, K}=H, \quad \lambda_{2, K}=h, \quad \mathbf{r}_{1, K}=\left(\begin{array}{l}
1 \\
0
\end{array}\right), \quad \mathbf{r}_{2, K}=\left(\begin{array}{l}
0 \\
1
\end{array}\right)
$$

(see Fig. 2.11).

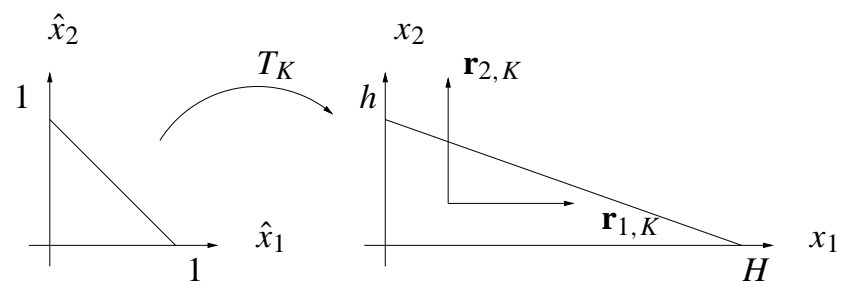

FIG. 2.1. A simple example of transformation from element $\hat{K}$ to $K$.

In the framework of anisotropic meshes, the classical minimum angle condition must be avoided. However, it is required that, for each vertex, the number of neighbouring vertices is bounded above, uniformly with respect to the mesh size $h$. Also, for any patch $\Delta_{K}$ (the set of triangles having a vertex common with $K$ ), the diameter of the corresponding reference patch $\Delta_{\widehat{K}}$, that is, $\Delta_{\widehat{K}}=$ $T_{K}^{-1}\left(\Delta_{K}\right)$, must be uniformly bounded independently of $h$. This latter hypothesis excludes some distorted patches (see Fig. 2.2.).

Let $I_{h}: H^{1}(\Omega) \rightarrow V_{h}$ be a Clément or Scott-Zhang like interpolation operator. We now recall some interpolation results due to [14,13]. 

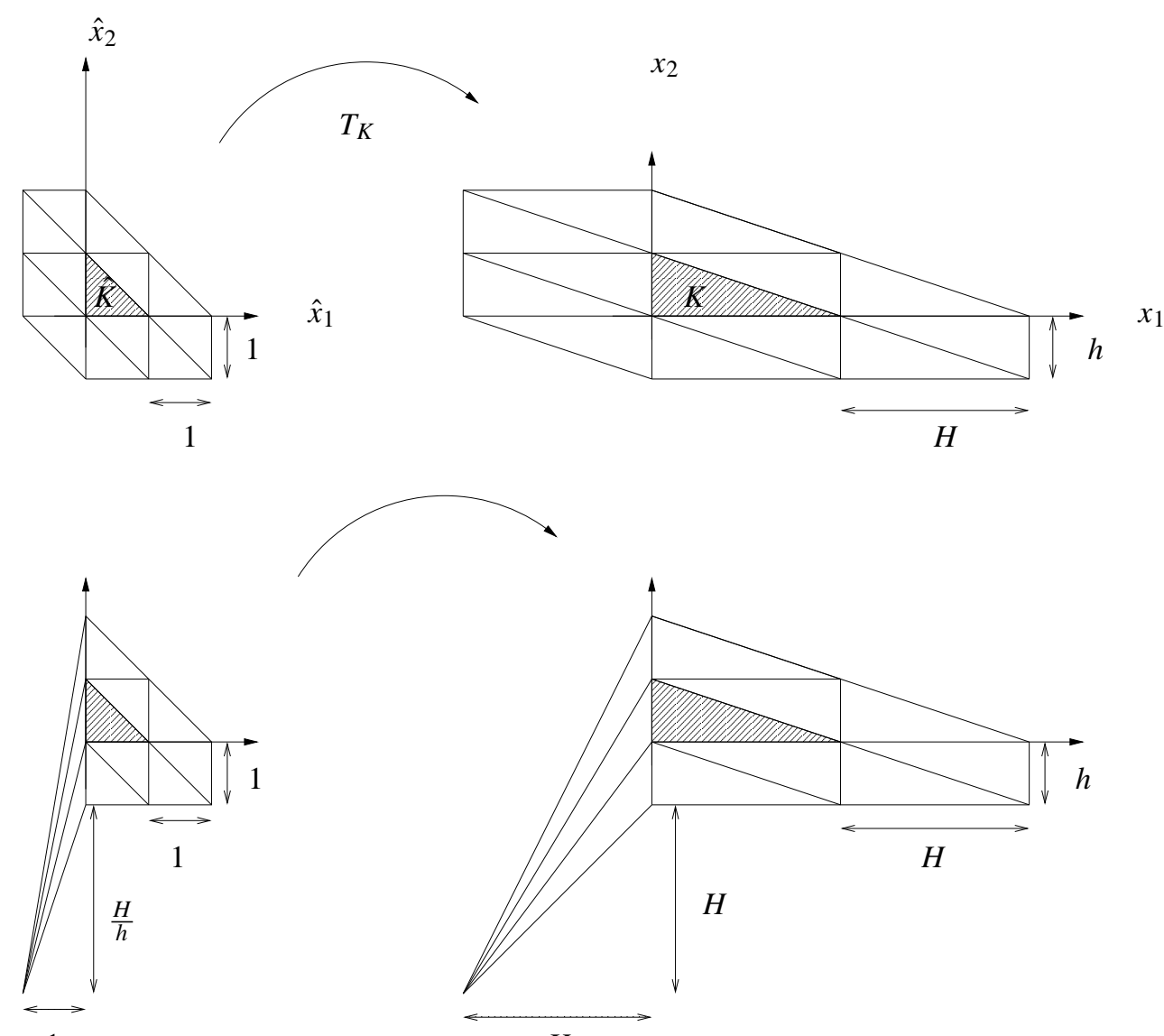

1

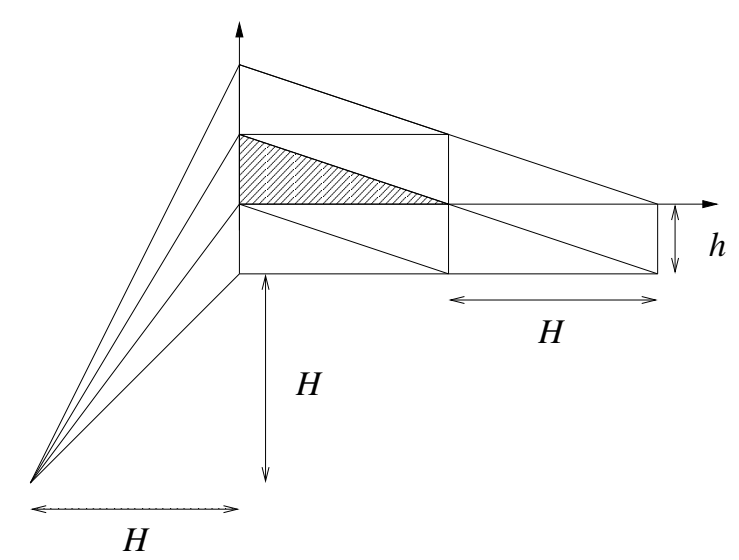

FIG. 2.2. Example of an acceptable patch (top): the size of the reference patch $\Delta_{\widehat{K}}$ does not depend on the aspect ratio $H / h$. Example of a nonacceptable patch (bottom): the size of the reference patch $\Delta_{\widehat{K}}$ now depends on the aspect ratio $H / h$.

Proposition 2.1 (Lemma 2.3 and Proposition 2.2 of [13]) There is a constant $C$ not depending on the mesh size nor on the mesh aspect ratio such that, for all $v \in H^{1}(\Omega)$, for all $K \in \mathcal{T}_{h}$, for all edges $e$ of $K$, we have

$$
\begin{aligned}
& \left\|v-I_{h} v\right\|_{L^{2}(K)} \leqslant C\left(\lambda_{1, K}^{2}\left(\mathbf{r}_{1, K}^{T} G_{K}(v) \mathbf{r}_{1, K}\right)+\lambda_{2, K}^{2}\left(\mathbf{r}_{2, K}^{T} G_{K}(v) \mathbf{r}_{2, K}\right)\right)^{1 / 2}, \\
& \left\|v-I_{h} v\right\|_{L^{2}(e)} \leqslant C h_{K}^{1 / 2}\left(\frac{\lambda_{1, K}}{\lambda_{2, K}}\left(\mathbf{r}_{1, K}^{T} G_{K}(v) \mathbf{r}_{1, K}\right)+\frac{\lambda_{2, K}}{\lambda_{1, K}}\left(\mathbf{r}_{2, K}^{T} G_{K}(v) \mathbf{r}_{2, K}\right)\right)^{1 / 2} .
\end{aligned}
$$

Here $G_{K}(v)$ denotes the $2 \times 2$ matrix defined by

$$
G_{K}(v)=\sum_{T \in \Delta_{K}}\left(\begin{array}{ll}
\int_{T}\left(\frac{\partial v}{\partial x_{1}}\right)^{2} \mathrm{~d} x & \int_{T} \frac{\partial v}{\partial x_{1}} \frac{\partial v}{\partial x_{2}} \mathrm{~d} x \\
\int_{T} \frac{\partial v}{\partial x_{1}} \frac{\partial v}{\partial x_{2}} \mathrm{~d} x & \int_{T}\left(\frac{\partial v}{\partial x_{2}}\right)^{2} \mathrm{~d} x
\end{array}\right)
$$




\section{Space discretization. Anisotropic a posteriori error estimates}

The semi-discrete finite element problem corresponding to discretization in space of 1.1 - -1.5 is the following. Assuming $\phi_{0}$ and $c_{0}$ to be continuous, we set $\phi_{h}(0)=r_{h} \phi_{0}, c_{h}(0)=r_{h} c_{0}$. For each $t \in[0, T]$ we find $\phi_{h}(t)$ and $c_{h}(t)$ in $V_{h}$ such that

$$
\begin{aligned}
& \int_{\Omega} \alpha \frac{\partial \phi_{h}}{\partial t} v_{h}+\int_{\Omega} A\left(\nabla \phi_{h}\right) \nabla \phi_{h} \cdot \nabla v_{h}-\int_{\Omega} S\left(c_{h}, \phi_{h}\right) v_{h}=0, \\
& \int_{\Omega} \frac{\partial c_{h}}{\partial t} w_{h}+\int_{\Omega} D_{1}\left(\phi_{h}\right) \nabla c_{h} \cdot \nabla w_{h}+\int_{\Omega} D_{2}\left(c_{h}, \phi_{h}\right) \nabla \phi_{h} \cdot \nabla w_{h}=0,
\end{aligned}
$$

for all $v_{h}, w_{h} \in V_{h}$. The well-posedness of the semi-discretized problem, for each fixed $h$, and for each $0 \leqslant \bar{a}<1$, follows by using estimates similar to those in $[8]$ and the fact that $\mid A\left(\nabla \phi_{h}\right) \nabla \phi_{h}-$ $A\left(\nabla \psi_{h}\right) \nabla \psi_{h}|\leqslant L| \nabla \phi_{h}-\nabla \psi_{h} \mid$ for all $\phi_{h}, \psi_{h}$ in $V_{h}$, which is proved in [7].

In order to derive sharp a posteriori error estimates, we need the following result, proved in [7], corresponding to strong ellipticity of $A(\cdot)$.

LEMma 3.1 Let $A(\cdot)$ be the operator defined by 1.6$)$ and let the convexity condition $\bar{a}<1 /\left(\kappa^{2}-1\right)$ hold. Then there exists $\mu$ (depending on $\bar{a}$ ) such that, for all $\phi, \psi \in H_{0}^{1}(\Omega)$, we have

$$
\mu\|\nabla(\phi-\psi)\|_{L^{2}(\Omega)}^{2} \leqslant(A(\nabla \phi) \nabla \phi-A(\nabla \psi) \nabla \psi, \nabla(\phi-\psi)) .
$$

We also need to assume that the error in the $L^{2}\left(0, T ; L^{2}(\Omega)\right)$ norm converges faster than the error in the $L^{2}\left(0, T ; H^{1}(\Omega)\right)$ norm, that is, there are two constants $C>0$ and $\left.\left.s \in\right] 0,1\right]$ such that for every mesh $\mathcal{T}_{h}$ we have

$$
\begin{aligned}
\int_{0}^{T}\left(\left\|\phi-\phi_{h}\right\|_{L^{2}(\Omega)}^{2}\right. & \left.+\left\|c-c_{h}\right\|_{L^{2}(\Omega)}^{2}\right) \\
& \leqslant C\left(\max _{K \in \mathcal{T}_{h}} \lambda_{2, K}\right)^{2 s} \int_{0}^{T}\left(\left\|\nabla\left(\phi-\phi_{h}\right)\right\|_{L^{2}(\Omega)}^{2}+\left\|\nabla\left(c-c_{h}\right)\right\|_{L^{2}(\Omega)}^{2}\right) .
\end{aligned}
$$

Let us comment on this assumption in the frame of isotropic meshes, that is to say, when $\lambda_{1, K}$ and $\lambda_{2, K}$ are of order $h$ for all $K \in \mathcal{T}_{h}$. In [7], an Euler scheme with continuous, piecewise linear finite elements is proposed for solving (3.1)- 3.2. A priori error estimates are obtained for isotropic meshes whenever $\bar{a}<1 /\left(\kappa^{2}-1\right)$. More precisely, assuming sufficient regularity of the solution, the error in the $L^{2}\left(0, T ; H^{1}(\Omega)\right)$ norm is shown to be $O(h+\tau), \tau$ being the time step. On the other hand, $O\left(h^{2}+\tau\right)$ convergence in the $L^{\infty}\left(0, T ; L^{2}(\Omega)\right)$ norm is proved for isotropic meshes in [16], but only in the case when $\bar{a}=0$. Therefore, we expect the following assumption:

$$
\int_{0}^{T}\left(\left\|\phi-\phi_{h}\right\|_{L^{2}(\Omega)}^{2}+\left\|c-c_{h}\right\|_{L^{2}(\Omega)}^{2}\right) \leqslant C h^{2 s} \int_{0}^{T}\left(\left\|\nabla\left(\phi-\phi_{h}\right)\right\|_{L^{2}(\Omega)}^{2}+\left\|\nabla\left(c-c_{h}\right)\right\|_{L^{2}(\Omega)}^{2}\right)
$$

to hold for isotropic meshes with $s=1$ when optimal convergence results hold in both $L^{2}\left(0, T ; H^{1}(\Omega)\right)$ and $L^{\infty}\left(0, T ; L^{2}(\Omega)\right)$ norms. Note that this assumption has been used in [17] to obtain a posteriori error estimates for isotropic finite elements and in the case when $\bar{a}=0$. When considering anisotropic meshes, $h$ in the above estimate should be replaced by $\max _{K \in \mathcal{T}_{h}} \lambda_{2, K}$, which yields (3.4). This assumption is checked numerically in Section 6 . 
The first step in deriving a posteriori error estimates consists in bounding the error by the equations residuals $R_{\phi}\left(\phi_{h}, c_{h}\right)$ and $R_{c}\left(\phi_{h}, c_{h}\right)$,

$$
R_{\phi}\left(\phi_{h}, c_{h}\right), R_{c}\left(\phi_{h}, c_{h}\right) \in\left(H^{1}(\Omega)\right)^{\prime} \quad \text { a.e. in }(0, T),
$$

defined by

$$
\begin{aligned}
\left\langle R_{\phi}\left(\phi_{h}, c_{h}\right), v\right\rangle & =\int_{\Omega}\left(\alpha \frac{\partial \phi_{h}}{\partial t} v+A\left(\nabla \phi_{h}\right) \nabla \phi_{h} \cdot \nabla v-S\left(c_{h}, \phi_{h}\right) v\right) \\
\left\langle R_{c}\left(\phi_{h}, c_{h}\right), v\right\rangle & =\int_{\Omega}\left(\frac{\partial c_{h}}{\partial t} v+D_{1}\left(\phi_{h}\right) \nabla c_{h} \cdot \nabla v+D_{2}\left(c_{h}, \phi_{h}\right) \nabla \phi_{h} \cdot \nabla v\right),
\end{aligned}
$$

for all $v \in H^{1}(\Omega)$, a.e. in $(0, T)$. Here $\langle\cdot, \cdot\rangle$ stands for the duality pairing between $H^{1}(\Omega)$ and its dual.

Proceeding as in [17], we have the following result.

Proposition 3.2 Let $\phi, c \in L^{2}\left(0, T ; H^{1}(\Omega)\right) \cap H^{1}\left(0, T ;\left(H^{1}(\Omega)\right)^{\prime}\right)$ be the weak solution of 1.1- 1.5 with $\bar{a}<\left(k^{2}-1\right)^{-1}$, let $\phi_{h}, c_{h}$ be the solution of $\sqrt{3.1}-\sqrt{3.2}$ and let $R_{\phi}\left(\phi_{h}, c_{h}\right)$, $R_{c}\left(\phi_{h}, c_{h}\right)$ be defined by $\left.\sqrt{3.5}-\sqrt{3.6}\right)$. Assume that $\phi, c \in L^{\infty}\left(0, T ; H^{2}(\Omega)\right)$ and define $M_{2}=$ $\left\|D_{2}\right\|_{L^{\infty}\left(\mathbb{R}^{2}\right)}$. Then there exists $h_{0}$ (depending on the constant $C$ in 3.4 and on the Lipschitz constants of $S, D_{1}$ and $D_{2}$ ) such that, for every mesh $\mathcal{T}_{h}$ satisfying $\max _{K \in \mathcal{T}_{h}} \lambda_{2, K} \leqslant h_{0}$, we have

$$
\begin{aligned}
\frac{\alpha}{2}\left\|\left(\phi-\phi_{h}\right)(T)\right\|_{L^{2}(\Omega)}^{2} & +\frac{\mu}{2} \int_{0}^{T}\left\|\nabla\left(\phi-\phi_{h}\right)\right\|_{L^{2}(\Omega)}^{2} \\
& +\frac{\mu D_{s}}{8 M_{2}^{2}}\left\|\left(c-c_{h}\right)(T)\right\|_{L^{2}(\Omega)}^{2}+\frac{\mu D_{s}^{2}}{8 M_{2}^{2}} \int_{0}^{T}\left\|\nabla\left(c-c_{h}\right)\right\|_{L^{2}(\Omega)}^{2} \\
\leqslant & \frac{\alpha}{2}\left\|\left(\phi-\phi_{h}\right)(0)\right\|_{L^{2}(\Omega)}^{2}+\frac{\mu D_{s}}{8 M_{2}^{2}}\left\|\left(c-c_{h}\right)(0)\right\|_{L^{2}(\Omega)}^{2} \\
& +\left|\int_{0}^{T}\left\langle R_{\phi}\left(\phi_{h}, c_{h}\right), \phi-\phi_{h}\right\rangle\right|+\frac{\mu D_{s}}{4 M_{2}^{2}}\left|\int_{0}^{T}\left\langle R_{c}\left(\phi_{h}, c_{h}\right), c-c_{h}\right\rangle\right| .
\end{aligned}
$$

Proof. Using the fact that $\phi$ is a weak solution of (1.1), we have

$$
\begin{aligned}
\left\langle\alpha \frac{\partial}{\partial t}\left(\phi-\phi_{h}\right), \phi-\phi_{h}\right\rangle & +\int_{\Omega}\left(A(\nabla \phi) \nabla \phi-A\left(\nabla \phi_{h}\right) \nabla \phi_{h}\right) \cdot \nabla\left(\phi-\phi_{h}\right) \\
= & \int_{\Omega}\left(S(c, \phi)-\alpha \frac{\partial \phi_{h}}{\partial t}\right)\left(\phi-\phi_{h}\right)-\int_{\Omega} A\left(\nabla \phi_{h}\right) \nabla \phi_{h} \cdot \nabla\left(\phi-\phi_{h}\right) .
\end{aligned}
$$

Integrating between $t=0$ and $t=T$, using the definition 3.5) and the strong ellipticity (3.3), we obtain

$$
\begin{aligned}
\frac{\alpha}{2}\left\|\left(\phi-\phi_{h}\right)(T)\right\|_{L^{2}(\Omega)}^{2}+\mu \int_{0}^{T} & \left\|\nabla\left(\phi-\phi_{h}\right)\right\|_{L^{2}(\Omega)}^{2} \\
\leqslant & \frac{\alpha}{2}\left\|\left(\phi-\phi_{h}\right)(0)\right\|_{L^{2}(\Omega)}^{2}+\left|\int_{0}^{T}\left\langle R_{\phi}\left(\phi_{h}, c_{h}\right), \phi-\phi_{h}\right\rangle\right| \\
& +\left|\int_{0}^{T} \int_{\Omega}\left(S(c, \phi)-S\left(c_{h}, \phi_{h}\right)\right)\left(\phi-\phi_{h}\right)\right| .
\end{aligned}
$$


On the other hand, using the fact that $c$ is a weak solution of (1.2), we have

$$
\begin{aligned}
\left\langle\frac{\partial}{\partial t}\left(c-c_{h}\right),\left(c-c_{h}\right)\right\rangle & +\int_{\Omega} D_{1}\left(\phi_{h}\right)\left|\nabla\left(c-c_{h}\right)\right|^{2} \\
= & \int_{\Omega}\left(-D_{2}(c, \phi) \nabla \phi \cdot \nabla\left(c-c_{h}\right)-\frac{\partial c_{h}}{\partial t}\left(c-c_{h}\right)\right) \\
& -\int_{\Omega} D_{1}\left(\phi_{h}\right) \nabla c_{h} \cdot \nabla\left(c-c_{h}\right)+\int_{\Omega}\left(D_{1}\left(\phi_{h}\right)-D_{1}(\phi)\right) \nabla c \cdot \nabla\left(c-c_{h}\right) .
\end{aligned}
$$

Thus, a time integration between $t=0$ and $t=T$, the fact that $D_{s} \leqslant D_{1}\left(\phi_{h}\right)$ and the use of definition (3.6) lead to

$$
\begin{aligned}
\frac{1}{2}\left\|\left(c-c_{h}\right)(T)\right\|_{L^{2}(\Omega)}^{2}+ & D_{s} \int_{0}^{T}\left\|\nabla\left(c-c_{h}\right)\right\|_{L^{2}(\Omega)}^{2} \\
\leqslant & \frac{1}{2}\left\|\left(c-c_{h}\right)(0)\right\|_{L^{2}(\Omega)}^{2}+\left|\int_{0}^{T}\left\langle R_{c}\left(\phi_{h}, c_{h}\right), c-c_{h}\right\rangle\right| \\
& +\left|\int_{0}^{T} \int_{\Omega}\left(D_{2}\left(c_{h}, \phi_{h}\right) \nabla \phi_{h}-D_{2}(c, \phi) \nabla \phi\right) \cdot \nabla\left(c-c_{h}\right)\right| \\
& +\left|\int_{0}^{T} \int_{\Omega}\left(D_{1}\left(\phi_{h}\right)-D_{1}(\phi)\right) \nabla c \cdot \nabla\left(c-c_{h}\right)\right| .
\end{aligned}
$$

Let $\beta$ be a positive number to be chosen later. Adding 3.8 and $\beta$ times 3.9 yields

$$
\begin{aligned}
\frac{\alpha}{2}\left\|\left(\phi-\phi_{h}\right)(T)\right\|_{L^{2}(\Omega)}^{2}+ & \mu \int_{0}^{T}\left\|\nabla\left(\phi-\phi_{h}\right)\right\|_{L^{2}(\Omega)}^{2} \\
& +\frac{\beta}{2}\left\|\left(c-c_{h}\right)(T)\right\|_{L^{2}(\Omega)}^{2}+\beta D_{s} \int_{0}^{T}\left\|\nabla\left(c-c_{h}\right)\right\|_{L^{2}(\Omega)}^{2} \\
\leqslant & \frac{\alpha}{2}\left\|\left(\phi-\phi_{h}\right)(0)\right\|_{L^{2}(\Omega)}^{2}+\frac{\beta}{2}\left\|\left(c-c_{h}\right)(0)\right\|_{L^{2}(\Omega)}^{2} \\
& +\left|\int_{0}^{T}\left\langle R_{\phi}\left(\phi_{h}, c_{h}\right), \phi-\phi_{h}\right\rangle\right|+\beta\left|\int_{0}^{T}\left\langle R_{c}\left(\phi_{h}, c_{h}\right), c-c_{h}\right\rangle\right| \\
& +\left|\int_{0}^{T} \int_{\Omega}\left(S(c, \phi)-S\left(c_{h}, \phi_{h}\right)\right)\left(\phi-\phi_{h}\right)\right| \\
& +\beta\left|\int_{0}^{T} \int_{\Omega}\left(D_{2}\left(c_{h}, \phi_{h}\right) \nabla \phi_{h}-D_{2}(c, \phi) \nabla \phi\right) \cdot \nabla\left(c-c_{h}\right)\right| \\
& +\beta\left|\int_{0}^{T} \int_{\Omega}\left(D_{1}\left(\phi_{h}\right)-D_{1}(\phi)\right) \nabla c \cdot \nabla\left(c-c_{h}\right)\right| .
\end{aligned}
$$

We now focus on the last three terms of 3.10. Since $S$ is Lipschitz there is a constant $C_{S}$ independent of $h$ such that

$$
\begin{aligned}
\mid \int_{0}^{T} \int_{\Omega}\left(S(c, \phi)-S\left(c_{h}, \phi_{h}\right)\right) & \left(\phi-\phi_{h}\right) \mid \\
& \leqslant C_{S} \int_{0}^{T}\left(\left\|c-c_{h}\right\|_{L^{2}(\Omega)}+\left\|\phi-\phi_{h}\right\|_{L^{2}(\Omega)}\right)\left\|\phi-\phi_{h}\right\|_{L^{2}(\Omega)} .
\end{aligned}
$$


Therefore, by [3.4), there is a constant $C_{S}$ independent of $h$ such that

$$
\begin{aligned}
\mid \int_{0}^{T} \int_{\Omega}(S(c, \phi) & \left.-S\left(c_{h}, \phi_{h}\right)\right)\left(\phi-\phi_{h}\right) \mid \\
& \leqslant C_{S}\left(\max _{K \in \mathcal{T}_{h}} \lambda_{2, K}\right)^{2 s} \int_{0}^{T}\left(\left\|\nabla\left(\phi-\phi_{h}\right)\right\|_{L^{2}(\Omega)}^{2}+\left\|\nabla\left(c-c_{h}\right)\right\|_{L^{2}(\Omega)}^{2}\right) .
\end{aligned}
$$

Let us now turn to the second of the last three terms of 3.10 . We have

$$
\begin{aligned}
& \left(D_{2}\left(c_{h}, \phi_{h}\right) \nabla \phi_{h}-D_{2}(c, \phi) \nabla \phi\right) \cdot \nabla\left(c-c_{h}\right) \\
& \quad=-\left(D_{2}(c, \phi)-D_{2}\left(c_{h}, \phi_{h}\right)\right) \nabla \phi \cdot \nabla\left(c-c_{h}\right)-D_{2}\left(c_{h}, \phi_{h}\right) \nabla\left(\phi-\phi_{h}\right) \cdot \nabla\left(c-c_{h}\right) .
\end{aligned}
$$

Since $D_{2}$ is bounded by $M_{2}$, using Young's inequality, we obtain

$$
\begin{aligned}
\mid \int_{0}^{T} \int_{\Omega} D_{2}\left(c_{h}, \phi_{h}\right) \nabla(\phi & \left.-\phi_{h}\right) \cdot \nabla\left(c-c_{h}\right) \mid \\
& \leqslant M_{2} \int_{0}^{T}\left(\frac{1}{2 \eta}\left\|\nabla\left(\phi-\phi_{h}\right)\right\|_{L^{2}(\Omega)}^{2}+\frac{\eta}{2}\left\|\nabla\left(c-c_{h}\right)\right\|_{L^{2}(\Omega)}^{2}\right),
\end{aligned}
$$

where $\eta>0$ will be chosen in what follows. On the other hand, using the Hölder inequality, we obtain

$$
\begin{aligned}
\mid \int_{0}^{T} \int_{\Omega}\left(D_{2}(c, \phi)-\right. & \left.D_{2}\left(c_{h}, \phi_{h}\right)\right) \nabla \phi \cdot \nabla\left(c-c_{h}\right) \mid \\
& \leqslant \int_{0}^{T}\|\nabla \phi\|_{L^{6}(\Omega)}\left\|D_{2}(c, \phi)-D_{2}\left(c_{h}, \phi_{h}\right)\right\|_{L^{3}(\Omega)}\left\|\nabla\left(c-c_{h}\right)\right\| .
\end{aligned}
$$

Since the imbedding of $H^{1}(\Omega)$ in $L^{6}(\Omega)$ is continuous and since by assumption $\phi \in$ $L^{\infty}\left(0, T ; H^{2}(\Omega)\right),\|\nabla \phi\|_{L^{6}(\Omega)}$ is bounded a.e. in $[0, T]$. Moreover, since $D_{2}$ is Lipschitz we have

$$
\left\|D_{2}(c, \phi)-D_{2}\left(c_{h}, \phi_{h}\right)\right\|_{L^{3}(\Omega)} \leqslant C\left(\left\|\phi-\phi_{h}\right\|_{L^{3}(\Omega)}+\left\|c-c_{h}\right\|_{L^{3}(\Omega)}\right) .
$$

Applying the above estimate in (3.13) yields

$$
\begin{aligned}
& \left|\int_{0}^{T} \int_{\Omega}\left(D_{2}(c, \phi)-D_{2}\left(c_{h}, \phi_{h}\right)\right) \nabla \phi \cdot \nabla\left(c-c_{h}\right)\right| \\
& \leqslant C \int_{0}^{T}\left(\left\|\phi-\phi_{h}\right\|_{L^{3}(\Omega)}+\left\|c-c_{h}\right\|_{L^{3}(\Omega)}\right)\left\|\nabla\left(c-c_{h}\right)\right\|_{L^{2}(\Omega)} .
\end{aligned}
$$

We now use a Gagliardo-Nirenberg inequality [6] to obtain

$$
\begin{aligned}
&\left|\int_{0}^{T} \int_{\Omega}\left(D_{2}(c, \phi)-D_{2}\left(c_{h}, \phi_{h}\right)\right) \nabla \phi \cdot \nabla\left(c-c_{h}\right)\right| \\
& \leqslant C \int_{0}^{T}\left(\left\|\phi-\phi_{h}\right\|_{H^{1}(\Omega)}^{1 / 2}\left\|\phi-\phi_{h}\right\|_{L^{2}(\Omega)}^{1 / 2}\right. \\
&\left.+\left\|c-c_{h}\right\|_{H^{1}(\Omega)}^{1 / 2}\left\|c-c_{h}\right\|_{L^{2}(\Omega)}^{1 / 2}\right)\left\|\nabla\left(c-c_{h}\right)\right\|_{L^{2}(\Omega)} .
\end{aligned}
$$


Finally, by repeated application of the Cauchy-Schwarz inequality and Young inequality, together with (3.4), we obtain

$$
\begin{aligned}
\mid \int_{0}^{T} \int_{\Omega}\left(D_{2}(c, \phi)\right. & \left.-D_{2}\left(c_{h}, \phi_{h}\right)\right) \nabla \phi \cdot \nabla\left(c-c_{h}\right) \mid \\
& \leqslant C\left(\max _{K \in \mathcal{T}_{h}} \lambda_{2, K}\right)^{s / 2} \int_{0}^{T}\left(\left\|\nabla\left(\phi-\phi_{h}\right)\right\|_{L^{2}(\Omega)}^{2}+\left\|\nabla\left(c-c_{h}\right)\right\|_{L^{2}(\Omega)}^{2}\right) .
\end{aligned}
$$

Let us now turn to the last term of 3.10. Since $D_{1}$ is Lipschitz we have, using the Hölder inequality,

$$
\begin{aligned}
& \left|\int_{0}^{T} \int_{\Omega}\left(D_{1}(\phi)-D_{1}\left(\phi_{h}\right)\right) \nabla c \cdot \nabla\left(c-c_{h}\right)\right| \\
& \quad \leqslant C \int_{0}^{T}\|\nabla c\|_{L^{6}(\Omega)}\left\|\phi-\phi_{h}\right\|_{L^{3}(\Omega)}\|\nabla(c-c)\|_{L^{2}(\Omega)} .
\end{aligned}
$$

Proceeding as for the previous term we obtain, using the fact that $c \in L^{\infty}\left(0, T ; H^{2}(\Omega)\right)$, a Gagliardo-Nirenberg inequality and 3.4,

$$
\begin{aligned}
\mid \int_{0}^{T} \int_{\Omega}\left(D_{1}(\phi)-\right. & \left.D_{1}\left(\phi_{h}\right)\right) \nabla c \cdot \nabla\left(c-c_{h}\right) \mid \\
& \leqslant C\left(\max _{K \in \mathcal{T}_{h}} \lambda_{2, K}\right)^{s / 2} \int_{0}^{T}\left(\left\|\nabla\left(\phi-\phi_{h}\right)\right\|_{L^{2}(\Omega)}^{2}+\left\|\nabla\left(c-c_{h}\right)\right\|_{L^{2}(\Omega)}^{2}\right) .
\end{aligned}
$$

Estimates 3.11, 3.14 and 3.15) applied in 3.10 yield

$$
\begin{aligned}
\frac{\alpha}{2}\left\|\left(\phi-\phi_{h}\right)(T)\right\|_{L^{2}(\Omega)}^{2}+ & \mu \int_{0}^{T}\left\|\nabla\left(\phi-\phi_{h}\right)\right\|_{L^{2}(\Omega)}^{2} \\
& +\frac{\beta}{2}\left\|\left(c-c_{h}\right)(T)\right\|_{L^{2}(\Omega)}^{2}+\beta D_{s} \int_{0}^{T}\left\|\nabla\left(c-c_{h}\right)\right\|_{L^{2}(\Omega)}^{2} \\
\leqslant & \frac{\alpha}{2}\left\|\left(\phi-\phi_{h}\right)(0)\right\|_{L^{2}(\Omega)}^{2}+\frac{\beta}{2}\left\|\left(c-c_{h}\right)(0)\right\|_{L^{2}(\Omega)}^{2} \\
& +\left|\int_{0}^{T}\left\langle R_{\phi}\left(\phi_{h}, c_{h}\right), \phi-\phi_{h}\right\rangle\right|+\beta\left|\int_{0}^{T}\left\langle R_{c}\left(\phi_{h}, c_{h}\right), c-c_{h}\right\rangle\right| \\
& +\beta M_{2} \int_{0}^{T}\left(\frac{1}{2 \eta}\left\|\nabla\left(\phi-\phi_{h}\right)\right\|_{L^{2}(\Omega)}^{2}+\frac{\eta}{2}\left\|\nabla\left(c-c_{h}\right)\right\|_{L^{2}(\Omega)}^{2}\right) \\
& +\tilde{C} \int_{0}^{T}\left(\left\|\nabla\left(\phi-\phi_{h}\right)\right\|_{L^{2}(\Omega)}^{2}+\left\|\nabla\left(c-c_{h}\right)\right\|_{L^{2}(\Omega)}^{2}\right)
\end{aligned}
$$

with $\tilde{C}=\left(C_{S}\left(\max _{K \in \mathcal{T}_{h}} \lambda_{2, K}\right)^{2 s}+C\left(\max _{K \in \mathcal{T}_{h}} \lambda_{2, K}\right)^{s / 2}\right), \beta, \eta>0$ and $C, C_{S}$ independent of $h$. We now choose $\beta$ and $\eta$ so that

$$
\mu-\frac{\beta M_{2}}{2 \eta}>0 \quad \text { and } \quad \beta D_{s}-\frac{\beta M_{2} \eta}{2}>0,
$$


for instance we can choose $\beta$ and $\eta$ such that

$$
\frac{\beta M_{2}}{2 \eta}=\frac{\mu}{4} \quad \text { and } \quad \frac{\beta M_{2} \eta}{2}=\frac{\beta D_{s}}{4},
$$

that is,

$$
\eta=\frac{D_{s}}{2 M_{2}} \quad \text { and } \quad \beta=\frac{\mu D_{s}}{4 M_{2}^{2}} .
$$

With this choice, 3.16 becomes

$$
\begin{aligned}
\frac{\alpha}{2}\left\|\left(\phi-\phi_{h}\right)(T)\right\|_{L^{2}(\Omega)}^{2} & +\frac{3 \mu}{4} \int_{0}^{T}\left\|\nabla\left(\phi-\phi_{h}\right)\right\|_{L^{2}(\Omega)}^{2} \\
& +\frac{\mu D_{s}}{8 M_{2}^{2}}\left\|\left(c-c_{h}\right)(T)\right\|_{L^{2}(\Omega)}^{2}+\frac{3 \mu D_{s}^{2}}{16 M_{2}^{2}} \int_{0}^{T}\left\|\nabla\left(c-c_{h}\right)\right\|_{L^{2}(\Omega)}^{2} \\
\leqslant & \frac{\alpha}{2}\left\|\left(\phi-\phi_{h}\right)(0)\right\|_{L^{2}(\Omega)}^{2}+\frac{\mu D_{s}}{8 M_{2}^{2}}\left\|\left(c-c_{h}\right)(0)\right\|_{L^{2}(\Omega)}^{2} \\
& +\left|\int_{0}^{T}\left\langle R_{\phi}\left(\phi_{h}, c_{h}\right), \phi-\phi_{h}\right\rangle\right|+\frac{\mu D_{s}}{4 M_{2}^{2}}\left|\int_{0}^{T}\left\langle R_{c}\left(\phi_{h}, c_{h}\right), c-c_{h}\right\rangle\right| \\
& +\tilde{C} \int_{0}^{T}\left(\left\|\nabla\left(\phi-\phi_{h}\right)\right\|_{L^{2}(\Omega)}^{2}+\left\|\nabla\left(c-c_{h}\right)\right\|_{L^{2}(\Omega)}^{2}\right) .
\end{aligned}
$$

Let $h_{0}$ be such that

$$
C_{S} h_{0}^{2 s}+C h_{0}^{s / 2}<\frac{3 \mu}{4} \quad \text { and } \quad C_{S} h_{0}^{2 s}+C h_{0}^{s / 2}<\frac{3 \mu D_{s}^{2}}{16 M_{2}^{2}} .
$$

For instance we can choose $h_{0}$ such that

$$
h_{0}=\min \left\{\left(\frac{K}{2 C_{S}}\right)^{1 /(2 s)},\left(\frac{K}{2 C}\right)^{2 / s}\right\}
$$

with

$$
K=\min \left(\frac{\mu}{4}, \frac{\mu D_{s}^{2}}{16 M_{2}^{2}}\right)
$$

and we obtain (3.7) for every mesh $\mathcal{T}_{h}$ such that $\max _{K \in \mathcal{T}_{h}} \lambda_{2, K} \leqslant h_{0}$. Keeping in mind that $C_{S}$ is inversely proportional to the interphase thickness squared we note that we can expect the dependence of $h_{0}$ on the interphase thickness to be linear when $s=1$.

Our goal now is to provide an upper bound for the equations residuals $R_{\phi}\left(\phi_{h}, c_{h}\right)$ and $R_{c}\left(\phi_{h}, c_{h}\right)$ defined in 3.5]-3.6. For this purpose, we proceed as in [18, 19] and introduce an anisotropic, explicit error estimator.

For each interior edge of $\mathcal{T}_{h}$, let us choose an arbitrary normal direction $\mathbf{n}$, and let [ $\left.\xi\right]$ denote the jump of $\xi$ across the edge. For each edge of $\mathcal{T}_{h}$ lying on the boundary $\partial \Omega$, we set [ $\left.\xi\right]$ to twice the inner side value of $\xi$. 
Proposition 3.3 Let $\phi_{h}, c_{h}$ be the solution of (3.1)- 3.2) and let $R_{\phi}\left(\phi_{h}, c_{h}\right), R_{c}\left(\phi_{h}, c_{h}\right)$ be defined by (3.5)-(3.6). For each triangle $K$ of the mesh, let $\lambda_{1, K}, \lambda_{2, K}, \mathbf{r}_{1, K}, \mathbf{r}_{2, K}$ and $G_{K}(\cdot)$ be defined as in Section 2 . Then there is a constant $C$ depending only on the interpolation constants of Proposition 2.1 such that for all $v \in L^{2}\left(0, T ; H^{1}(\Omega)\right)$ we have, using for simplicity the notation $S=S\left(c_{h}, \phi_{h}\right), D_{1}=D_{1}\left(\phi_{h}\right), D_{2}=D_{2}\left(c_{h}, \phi_{h}\right)$,

$$
\begin{aligned}
& \left|\int_{0}^{T}\left\langle R_{\phi}\left(\phi_{h}, c_{h}\right), v\right\rangle\right| \leqslant C \int_{0}^{T} \sum_{K \in \mathcal{T}_{h}}\left(\left\|\alpha \frac{\partial \phi_{h}}{\partial t}-\operatorname{div}\left(A\left(\nabla \phi_{h}\right) \nabla \phi_{h}\right)-S\right\|_{L^{2}(K)}\right. \\
& \left.+\frac{1}{2 \lambda_{2, K}^{1 / 2}}\left\|\left[A\left(\nabla \phi_{h}\right) \nabla \phi_{h} \cdot \mathbf{n}\right]\right\|_{L^{2}(\partial K)}\right) \\
& \times\left(\lambda_{1, K}^{2}\left(\mathbf{r}_{1, K}^{T} G_{K}(v) \mathbf{r}_{1, K}\right)+\lambda_{2, K}^{2}\left(\mathbf{r}_{2, K}^{T} G_{K}(v) \mathbf{r}_{2, K}\right)\right)^{1 / 2}, \\
& \left|\int_{0}^{T}\left\langle R_{c}\left(\phi_{h}, c_{h}\right), v\right\rangle\right| \leqslant C \int_{0}^{T} \sum_{K \in \mathcal{T}_{h}}\left(\left\|\frac{\partial c_{h}}{\partial t}-\operatorname{div}\left(D_{1} \nabla c_{h}+D_{2} \nabla \phi_{h}\right)\right\|_{L^{2}(K)}\right. \\
& \left.+\frac{1}{2 \lambda_{2, K}^{1 / 2}}\left\|\left[D_{1} \frac{\partial c_{h}}{\partial n}+D_{2} \frac{\partial \phi_{h}}{\partial n}\right]\right\|_{L^{2}(\partial K)}\right) \\
& \times\left(\lambda_{1, K}^{2}\left(\mathbf{r}_{1, K}^{T} G_{K}(v) \mathbf{r}_{1, K}\right)+\lambda_{2, K}^{2}\left(\mathbf{r}_{2, K}^{T} G_{K}(v) \mathbf{r}_{2, K}\right)\right)^{1 / 2} .
\end{aligned}
$$

Proof. We will sketch the proof for the first estimate only. The second estimate follows in the same manner. Let $v$ be an element of $L^{2}\left(0, T ; H^{1}(\Omega)\right)$. Using (3.1) and 3.5 we have

$$
\left\langle R_{\phi}\left(\phi_{h}, c_{h}\right), v\right\rangle=\left\langle R_{\phi}\left(\phi_{h}, c_{h}\right), v-v_{h}\right\rangle \quad \forall v_{h} \in V_{h} \quad \text { a.e. in }(0, T) .
$$

From the definition of $R_{\phi}\left(\phi_{h}, c_{h}\right)$ we have

$$
\left\langle R_{\phi}\left(\phi_{h}, c_{h}\right), v-v_{h}\right\rangle=\sum_{K \in \mathcal{T}_{h}}\left\{\int_{K}\left(\frac{\partial \phi_{h}}{\partial t}-S\left(c_{h}, \phi_{h}\right)\right)\left(v-v_{h}\right)+\int_{K} A\left(\nabla \phi_{h}\right) \nabla \phi_{h} \cdot \nabla\left(v-v_{h}\right)\right\} .
$$

Integrating by parts in the last term we obtain

$$
\begin{aligned}
\left\langle R_{\phi}\left(\phi_{h}, c_{h}\right), v-v_{h}\right\rangle=\sum_{K \in \mathcal{T}_{h}}\{ & \int_{K}\left(\frac{\partial \phi_{h}}{\partial t}-\operatorname{div}\left(A\left(\nabla \phi_{h}\right) \nabla \phi_{h}\right)-S\left(c_{h}, \phi_{h}\right)\right)\left(v-v_{h}\right) \\
& \left.+\frac{1}{2} \int_{\partial K}\left[A\left(\nabla \phi_{h}\right) \nabla \phi_{h} \cdot \mathbf{n}\right]\left(v-v_{h}\right)\right\} .
\end{aligned}
$$

Thus, the Cauchy-Schwarz inequality yields

$$
\begin{gathered}
\left|\left\langle R_{\phi}\left(\phi_{h}, c_{h}\right), v-v_{h}\right\rangle\right| \\
\leqslant \sum_{K \in \mathcal{T}_{h}}\left\{\left\|\frac{\partial \phi_{h}}{\partial t}-\operatorname{div}\left(A\left(\nabla \phi_{h}\right) \nabla \phi_{h}\right)-S\left(c_{h}, \phi_{h}\right)\right\|_{L^{2}(K)}\left\|v-v_{h}\right\|_{L^{2}(K)}\right. \\
\left.\quad+\frac{1}{2}\left\|\left[A\left(\nabla \phi_{h}\right) \nabla \phi_{h} \cdot \mathbf{n}\right]\right\|_{L^{2}(\partial K)}\left\|v-v_{h}\right\|_{L^{2}(\partial K)}\right\} .
\end{gathered}
$$


It then suffices to choose $v_{h}=I_{h} v$, where $I_{h}$ is Clément's interpolant, to use the interpolation estimates of Proposition 2.1 and the fact that

$$
\lambda_{2, K} h_{\hat{K}} \leqslant h_{K} \leqslant \lambda_{1, K} h_{\hat{K}}
$$

to conclude.

Putting together the results of Propositions 3.2 and 3.3 , we obtain the main theoretical result of this paper.

THEOREM 3.4 Let $\phi, c$ be the weak solution of (1.1)-(1.5), and let $\phi_{h}, c_{h}$ be the solution of (3.1)3.2. Assume that $\phi, c \in L^{\infty}\left(0, T ; H^{2}(\Omega)\right)$ and define $M_{2}=\left\|D_{2}\right\|_{L^{\infty}\left(\mathbb{R}^{2}\right)}$. For each triangle $K$ of the mesh, let $\lambda_{1, K}, \lambda_{2, K}, \mathbf{r}_{1, K}, \mathbf{r}_{2, K}$ and $G_{K}(\cdot)$ be defined as in Section 2. Then there is a constant $C$ depending only on the interpolation constants of Proposition 2.1 such that for every mesh $\mathcal{T}_{h}$ with $\max _{K \in \mathcal{T}_{h}} \lambda_{2, K}$ sufficiently small, we have

$$
\begin{aligned}
\frac{\alpha}{2}\left\|\left(\phi-\phi_{h}\right)(T)\right\|_{L^{2}(\Omega)}^{2}+\frac{\mu}{2} \int_{0}^{T}\left\|\nabla\left(\phi-\phi_{h}\right)\right\|_{L^{2}(\Omega)}^{2} \\
+\frac{\mu D_{s}}{8 M_{2}^{2}}\left\|\left(c-c_{h}\right)(T)\right\|_{L^{2}(\Omega)}^{2}+\frac{\mu D_{s}^{2}}{8 M_{2}^{2}} \int_{0}^{T}\left\|\nabla\left(c-c_{h}\right)\right\|_{L^{2}(\Omega)}^{2} \\
\leqslant \frac{\alpha}{2}\left\|\left(\phi-\phi_{h}\right)(0)\right\|_{L^{2}(\Omega)}^{2}+\frac{\mu D_{s}}{8 M_{2}^{2}}\left\|\left(c-c_{h}\right)(0)\right\|_{L^{2}(\Omega)}^{2} \\
+C \int_{0}^{T} \sum_{K \in \mathcal{T}_{h}}\left(\left\|\alpha \frac{\partial \phi_{h}}{\partial t}-\operatorname{div}\left(A\left(\nabla \phi_{h}\right) \nabla \phi_{h}\right)-S\left(c_{h}, \phi_{h}\right)\right\|_{L^{2}(K)}\right. \\
\left.+\frac{1}{2 \lambda_{2, K}^{1 / 2}}\left\|\left[A\left(\nabla \phi_{h}\right) \nabla \phi_{h} \cdot \mathbf{n}\right]\right\|_{L^{2}(\partial K)}\right) \\
\times\left(\lambda_{1, K}^{2}\left(\mathbf{r}_{1, K}^{T} G_{K}\left(\phi-\phi_{h}\right) \mathbf{r}_{1, K}\right)+\lambda_{2, K}^{2}\left(\mathbf{r}_{2, K}^{T} G_{K}\left(\phi-\phi_{h}\right) \mathbf{r}_{2, K}\right)\right)^{1 / 2} \\
+C \frac{\mu D_{s}^{2}}{4 M_{2}^{2}} \int_{0}^{T} \sum_{K \in \mathcal{T}_{h}}\left(\left\|\frac{\partial c_{h}}{\partial t}-\operatorname{div}\left(D_{1}\left(\phi_{h}\right) \nabla c_{h}+D_{2}\left(c_{h}, \phi_{h}\right) \nabla \phi_{h}\right)\right\|_{L^{2}(K)}\right. \\
\left.+\frac{1}{2 \lambda_{2, K}^{1 / 2}}\left\|\left[D_{1}\left(\phi_{h}\right) \frac{\partial c_{h}}{\partial n}+D_{2}\left(c_{h}, \phi_{h}\right) \frac{\partial \phi_{h}}{\partial n}\right]\right\|_{L^{2}(\partial K)}\right) \\
\times\left(\lambda_{1, K}^{2}\left(\mathbf{r}_{1, K}^{T} G_{K}\left(c-c_{h}\right) \mathbf{r}_{1, K}\right)+\lambda_{2, K}^{2}\left(\mathbf{r}_{2, K}^{T} G_{K}\left(c-c_{h}\right) \mathbf{r}_{2, K}\right)\right)^{1 / 2} .
\end{aligned}
$$

Estimate 3.20 is not a usual a posteriori error estimate since $\phi$ and $c$ are still involved in the right hand side. We then proceed as in [18, 19] and introduce an estimator based on superconvergent recovery, namely a Zienkiewicz-Zhu (Z-Z) like estimator [24,3,25]. More precisely, we consider the simplest Z-Z error estimator as defined in [21,1]. For instance, the Z-Z error estimator corresponding to $\nabla\left(\phi-\phi_{h}\right)$ is defined by the difference between $\nabla \phi_{h}$ and an approximate $L^{2}$ projection of $\nabla \phi_{h}$ onto $V_{h}^{2}$, namely:

$$
\boldsymbol{\eta}^{Z Z}\left(\phi_{h}\right)=\left(\begin{array}{l}
\eta_{1}^{Z Z}\left(\phi_{h}\right) \\
\eta_{2}^{Z Z}\left(\phi_{h}\right)
\end{array}\right)=\left(\begin{array}{c}
\left(I-\Pi_{h}\right)\left(\frac{\partial \phi_{h}}{\partial x_{1}}\right) \\
\left(I-\Pi_{h}\right)\left(\frac{\partial \phi_{h}}{\partial x_{2}}\right)
\end{array}\right) .
$$


Here $\Pi_{h}: g \in L^{2}(\Omega) \rightarrow \Pi_{h} g \in V_{h}$ is defined by

$$
\int_{\Omega} r_{h}\left(\left(\Pi_{h} g\right) \cdot \mathbf{v}_{h}\right)=\int_{\Omega} g \cdot \mathbf{v}_{h} \quad \forall \mathbf{v}_{h} \in V_{h},
$$

where $r_{h}$ denotes the usual Lagrange interpolant. In other words, from constant values of $\nabla \phi_{h}$ on triangles, we build values at vertices $P$ using the formula

$$
\left(\begin{array}{l}
\Pi_{h}\left(\frac{\partial \phi_{h}}{\partial x_{1}}\right)(P) \\
\Pi_{h}\left(\frac{\partial \phi_{h}}{\partial x_{2}}\right)(P)
\end{array}\right)=\frac{1}{\sum_{\substack{P \in K \\
K \in \mathcal{T}_{h}}}|K|}\left(\begin{array}{l}
\sum_{\substack{P \in K \\
K \in \mathcal{T}_{h}}}|K|\left(\frac{\partial \phi_{h}}{\partial x_{1}}\right)_{\mid K} \\
\sum_{\substack{P \in K \\
K \in \mathcal{T}_{h}}}|K|\left(\frac{\partial \phi_{h}}{\partial x_{2}}\right)_{\mid K}
\end{array}\right) .
$$

From [2, 4, 21] we know that for a certain class of meshes (namely parallel meshes) and for smooth solutions, Z-Z like error estimators are asymptotically exact. Our anisotropic error indicator corresponding to $\phi-\phi_{h}$ is obtained by replacing the matrix $G_{K}\left(\phi-\phi_{h}\right)$ in 3.20 by the matrix $\tilde{G}_{K}\left(\phi_{h}\right)$ defined by

$$
\tilde{G}_{K}\left(\phi_{h}\right)=\left(\begin{array}{cc}
\int_{K}\left(\eta_{1}^{Z Z}\left(\phi_{h}\right)\right)^{2} \mathrm{~d} x & \int_{K} \eta_{1}^{Z Z}\left(\phi_{h}\right) \eta_{2}^{Z Z}\left(\phi_{h}\right) \mathrm{d} x \\
\int_{K} \eta_{1}^{Z Z}\left(\phi_{h}\right) \eta_{2}^{Z Z}\left(\phi_{h}\right) \mathrm{d} x & \int_{K}\left(\eta_{2}^{Z Z}\left(\phi_{h}\right)\right)^{2} \mathrm{~d} x
\end{array}\right) .
$$

Finally, our anisotropic error indicator corresponding to $\phi-\phi_{h}$ is defined on each triangle $K$ by

$$
\begin{aligned}
& \int_{0}^{T} \sum_{K \in \mathcal{T}_{h}}\left(\| \alpha \frac{\partial \phi_{h}}{\partial t}-\operatorname{div}(\right.\left.A\left(\nabla \phi_{h}\right) \nabla \phi_{h}\right)- \\
& S\left(c_{h}, \phi_{h}\right) \|_{L^{2}(K)} \\
&\left.+\frac{1}{2 \lambda_{2, K}^{1 / 2}}\left\|\left[A\left(\nabla \phi_{h}\right) \nabla \phi_{h} \cdot \mathbf{n}\right]\right\|_{L^{2}(\partial K)}\right) \\
& \times\left(\lambda_{1, K}^{2}\left(\mathbf{r}_{1, K}^{T} \tilde{G}_{K}\left(\phi_{h}\right) \mathbf{r}_{1, K}\right)+\lambda_{2, K}^{2}\left(\mathbf{r}_{2, K}^{T} \tilde{G}_{K}\left(\phi_{h}\right) \mathbf{r}_{2, K}\right)\right)^{1 / 2} .
\end{aligned}
$$

Accordingly, our anisotropic error indicator corresponding to $c-c_{h}$ is defined on each triangle $K$ by

$$
\begin{aligned}
\int_{0}^{T} \sum_{K \in \mathcal{T}_{h}}\left(\left\|\frac{\partial c_{h}}{\partial t}-\operatorname{div}\left(D_{1}\left(\phi_{h}\right) \nabla c_{h}+D_{2}\left(c_{h}, \phi_{h}\right) \nabla \phi_{h}\right)\right\|_{L^{2}(K)}\right. \\
\left.+\frac{1}{2 \lambda_{2, K}^{1 / 2}}\left\|\left[D_{1}\left(\phi_{h}\right) \frac{\partial c_{h}}{\partial n}+D_{2}\left(c_{h}, \phi_{h}\right) \frac{\partial \phi_{h}}{\partial n}\right]\right\|_{L^{2}(\partial K)}\right) \\
\times\left(\lambda_{1, K}^{2}\left(\mathbf{r}_{1, K}^{T} \tilde{G}_{K}\left(c_{h}\right) \mathbf{r}_{1, K}\right)+\lambda_{2, K}^{2}\left(\mathbf{r}_{2, K}^{T} \tilde{G}_{K}\left(c_{h}\right) \mathbf{r}_{2, K}\right)\right)^{1 / 2} .
\end{aligned}
$$

\section{Time discretization}

Given an integer $N$, we set $\tau=T / N$ the time step, $t^{n}=n \tau, n=0, \ldots, N$. Assuming the initial data $\phi_{0}$ and $c_{0}$ to be continuous, we set $\phi_{h}^{0}=r_{h} \phi_{0}, c_{h}^{0}=r_{h} c_{0}$, where $r_{h}$ is the classical Lagrange interpolant. Then, for each $n=1, \ldots, N$, we find $\phi_{h}^{n}$ in $V_{h}$ such that 


$$
\begin{aligned}
\int_{\Omega} \alpha \frac{\phi_{h}^{n}-\phi_{h}^{n-1}}{\tau} v_{h}+ & \int_{\Omega} A\left(\nabla \phi_{h}^{n-1}\right) \nabla \phi_{h}^{n} \cdot \nabla v_{h} \\
& -\int_{\Omega}\left(S\left(c_{h}^{n-1}, \phi_{h}^{n-1}\right)+\frac{\partial S}{\partial \phi}\left(c_{h}^{n-1}, \phi_{h}^{n-1}\right)\left(\phi_{h}^{n}-\phi_{h}^{n-1}\right)\right) v_{h}=0
\end{aligned}
$$

for all $v_{h} \in V_{h}$. Then we find $c_{h}^{n}$ in $V_{h}$ such that

$$
\int_{\Omega} \frac{c_{h}^{n}-c_{h}^{n-1}}{\tau} w_{h}+\int_{\Omega} D_{1}\left(\phi_{h}^{n}\right) \nabla c_{h}^{n} \cdot \nabla w_{h}+\int_{\Omega} D_{2}\left(c_{h}^{n-1}, \phi_{h}^{n}\right) \nabla \phi_{h}^{n} \cdot \nabla w_{h}=0
$$

for all $v_{h} \in V_{h}$.

Equation (4.1) corresponds to an implicit Euler discretization of (3.1), with only one Newton step at each time step. Let $C \geqslant 0$ be such that $-C \leqslant \frac{\partial S}{\partial \phi}(c, \phi)$ for all $c, \phi \in \mathbb{R}^{2}$. Then problem (4.1) is well-posed for all $0 \leqslant \bar{a}<1$ when $\tau \leqslant \alpha / C$ (by the Lax-Milgram lemma). This limitation prevents the use of large time steps and is well known in the framework of mean curvature flows [11, 12].

\section{An adaptive algorithm}

We now propose an adaptive algorithm, the time step $\tau$ being fixed. The goal is to build anisotropic triangulations $\mathcal{T}_{h}^{n}, n=1, \ldots, N$, such that the relative estimated error in the $L^{2}\left(0, T ; H^{1}(\Omega)\right)$ norm is close to a preset tolerance TOL. Proceeding as in [19] we introduce $c_{h \tau}$, the continuous, piecewise linear approximation in time defined by

$$
c_{h \tau}(x, t)=\frac{t-t^{n-1}}{\tau} c_{h}^{n}(x)+\frac{t^{n}-t}{\tau} c_{h}^{n-1}(x), \quad t^{n-1} \leqslant t \leqslant t^{n}, x \in \Omega .
$$

Then our adaptive algorithm aims at building anisotropic triangulations $\mathcal{T}_{h}^{n}, n=1, \ldots, N$, such that

$$
0.75 \mathrm{TOL} \leqslant \frac{\sum_{n=1}^{N} \sum_{K \in \mathcal{T}_{h}}\left(\eta_{n, K}\left(c_{h \tau}\right)\right)^{2}}{\int_{0}^{T} \int_{\Omega}\left|\nabla c_{h \tau}\right|^{2}} \leqslant 1.25 \text { TOL. }
$$

Here $\eta_{n, K}\left(c_{h \tau}\right)$ is the simplified error indicator corresponding to 3.24 , defined on $\left[t^{n-1}, t^{n}\right] \times K$ by

$$
\begin{aligned}
\left(\eta_{n, K}\left(c_{h \tau}\right)\right)^{2}= & \int_{t^{n-1}}^{t^{n}} \frac{1}{2 \lambda_{2, K}^{1 / 2}}\left\|\left[\frac{\partial c_{h \tau}}{\partial n}\right]\right\|_{L^{2}(\partial K)} \\
& \times\left(\lambda_{1, K}^{2}\left(\mathbf{r}_{1, K}^{T} \tilde{G}_{K}\left(c_{h \tau}\right) \mathbf{r}_{1, K}\right)+\lambda_{2, K}^{2}\left(\mathbf{r}_{2, K}^{T} \tilde{G}_{K}\left(c_{h \tau}\right) \mathbf{r}_{2, K}\right)\right)^{1 / 2}
\end{aligned}
$$

where $\tilde{G}_{K}\left(c_{h \tau}\right)$ is defined as in 3.22. A sufficient condition to satisfy 5.2 is to build, for each $n=1, \ldots, N$, an anisotropic triangulation $\mathcal{T}_{h}^{n}$ such that

$$
\frac{0.75^{2} \mathrm{TOL}^{2}}{\mathrm{NV}_{h}^{n}} \int_{t^{n-1}}^{t^{n}} \int_{\Omega}\left|\nabla c_{h \tau}\right|^{2} \leqslant\left(\eta_{n, K}\left(c_{h \tau}\right)\right)^{2} \leqslant \frac{1.25^{2} \mathrm{TOL}^{2}}{\mathrm{NV}_{h}^{n}} \int_{t^{n-1}}^{t^{n}} \int_{\Omega}\left|\nabla c_{h \tau}\right|^{2}
$$

for all triangles $K \in \mathcal{T}_{h}^{n}$, where $\mathrm{NV}_{h}^{n}$ is the number of vertices of the mesh $\mathcal{T}_{h}^{n}$. We then proceed as in [18, 19] to build such an anisotropic mesh, using the BL2D mesh generator [5]. 

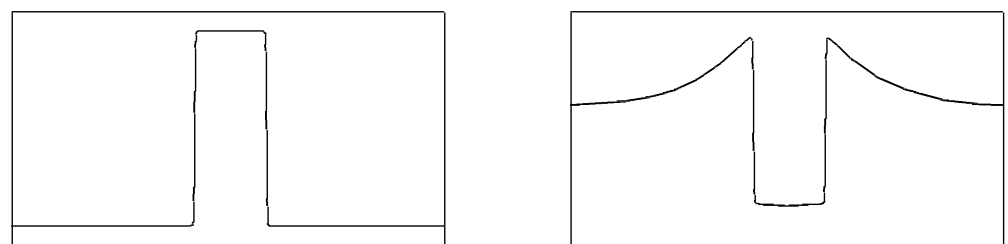

FIG. 5.1. Typical profiles of the phase field (left) and concentration field (right). The phase field has values zero or one, except in the phase change region. The concentration field changes rapidly across the phase change region, but may also vary outside the phase change region.

The reasons for using the simplified error indicator (5.3) instead of $(3.23)$ and $(3.24)$ are the following. First, we have disregarded the error estimator related to the phase field $\phi$ because, when computing solutal dendrites, although both $\phi$ and $c$ vary strongly in the small region corresponding to the solid-liquid interface, the function $c$ may also vary in other regions, whereas $\phi$ does not (see Fig. 5.1. Secondly, in order to simplify the implementation of the error indicator, we have kept only the jump of the gradients across the edge, which is the generic term in explicit error estimators. Theoretical arguments for such a choice in the frame of elliptic problems can be found in [9].

\section{Numerical results}

We consider the model of [22], the notations being those of [15]. The source term $S$ in (1.1] is defined by

$$
S(c, \phi)=-\frac{1}{\lambda^{2}} \phi(1-\phi)(1-2 \phi)+\frac{5 m_{l}}{\lambda \Gamma} \phi^{2}(1-\phi)^{2}\left(\frac{c}{1-\phi+k \phi}-c_{l}\right) \quad \text { if } 0 \leqslant \phi \leqslant 1,
$$

whereas $S(c, \phi)=0$ if $\phi<0$ or $\phi>1$. Here $\lambda$ is the thickness of the solid-liquid interface, $m_{l}$ is the liquidus slope in the phase diagram, $\Gamma$ is the Gibbs-Thomson isotropic coefficient, $c_{l}$ is the liquid concentration in the phase diagram, and $k$ is the phase diagram partition coefficient (thus $c_{s}=k c_{l}$, where $c_{s}$ is the solid concentration in the phase diagram). The coefficient $\alpha$ in $(1.1)$ equals $1 /\left(\mu_{k} \Gamma\right)$, where $\mu_{k}$ is the interface kinetic coefficient. The first term in the definition of $S(\cdot, \cdot)$ is nothing but the derivative of a double-well potential that forces $\phi$ to have values zero or one, except in the phase change region. In the limit when $\lambda$ goes to zero, the second term in the definition of $S(\cdot, \cdot)$ links the normal velocity of the solid-liquid interface, some anisotropic measure of the interface curvature, and the concentration field. The function $D_{1}$ in $(1.2)$ is given by

$$
D_{1}(\phi)=D_{s}+\frac{1-\phi}{1-\phi+k \phi}\left(D_{l}-D_{s}\right) \quad \text { if } 0 \leqslant \phi \leqslant 1,
$$

whereas $D_{1}(\phi)=D_{l}$ if $\phi<0$ and $D_{1}(\phi)=D_{s}$ if $1<\phi$. Here $D_{s}$ and $D_{l}$ are the solid and liquid diffusion coefficients. Finally, the function $D_{2}$ in $(1.2)$ is given by

$$
D_{2}(c, \phi)=D_{1}(\phi) \frac{(1-k) c(1-c)}{1-\phi+k \phi} \quad \text { if } 0 \leqslant c \leqslant 1,
$$

whereas $D_{2}(c, \phi)=0$ if $c<0$ or $c>1$. All the physical parameters are given below in the international MKSA unit system. 


\subsection{A simple test case with exact solution}

Our first goal is to validate numerically assumption (3.4) in the frame of anisotropic meshes. For this purpose, we set the computational domain to $\Omega=[-0.0002,0.0002]^{2}$ and we add source terms in (1.1)-1.2 so that $\phi$ and $c$ are given by

$$
\phi\left(x_{1}, x_{2}, t\right)=c\left(x_{1}, x_{2}, t\right)=\frac{1-\tanh \left(\left(x_{1}-v t\right) / \delta\right)}{2},
$$

where $v=2 \cdot 10^{-4}$ and $\delta=10^{-5}$. Dirichlet boundary conditions are prescribed on the vertical sides of $\Omega$, and homogeneous Neumann boundary conditions on the horizontal sides. The isolines of $\phi$ and $c$ at time $t=0$ and $t=0.5$ are shown in Fig. 6.1. The physical parameters $D_{1}$ and $D_{2}$ involved in the definition of $S$ are given in Table 6.1 the time step is $\tau=5 \cdot 10^{-5}$ and is small enough so that the error due to time discretization is negligible. Meshes with strong anisotropy are used to validate assumption (3.4), an example of mesh being shown in Fig. 6.1.

TABLE 6.1

Test case with exact solution: parameters used for the computations

\begin{tabular}{cccccccc}
\hline$\lambda$ & $m_{l}$ & $\Gamma$ & $c_{s}$ & $c_{l}$ & $D_{s}$ & $D_{l}$ & $\mu_{k}$ \\
\hline $10^{-5}$ & -260 & 0.1 & 0.015 & 0.0238 & $5 \cdot 10^{-10}$ & $5 \cdot 10^{-9}$ & 0.0015 \\
\hline
\end{tabular}

TABLE 6.2

Various convergence results for the travelling wave solution

Anisotropic meshes refined in both horizontal and vertical directions

\begin{tabular}{ccccc}
\hline$h 1-h 2$ & $e_{L^{2}}$ & $e_{H^{1}}$ & $e i^{Z Z}$ & $e i^{A}$ \\
\hline $0.000005-0.0001$ & $1.1 \cdot 10^{-6}$ & 0.29 & 1.01 & 1.85 \\
\hline $0.0000025-0.00005$ & $3.2 \cdot 10^{-7}$ & 0.13 & 1.01 & 1.74 \\
\hline $0.00000125-0.000025$ & $8.8 \cdot 10^{-8}$ & 0.066 & 1.01 & 1.74 \\
\hline Anisotropic meshes refined in horizontal direction only \\
\hline$h 1-h 2$ & $e_{L^{2}}$ & $e_{H^{1}}$ & $e i^{Z Z}$ & $e i^{A}$ \\
\hline $0.000005-0.0001$ & $1.1 \cdot 10^{-6}$ & 0.29 & 1.01 & 1.85 \\
\hline $0.0000025-0.0001$ & $4.4 \cdot 10^{-7}$ & 0.14 & 1.00 & 1.79 \\
\hline $0.00000125-0.0001$ & $1.2 \cdot 10^{-8}$ & 0.061 & 1.00 & 1.79 \\
\hline Anisotropic meshes refined in vertical direction only \\
\hline$h 1-h 2$ & $e_{L^{2}}$ & $e_{H^{1}}$ & $e i^{Z Z}$ & $e i^{A}$ \\
\hline $0.00005-0.00001$ & $4.0 \cdot 10^{-5}$ & 4.3 & 0.85 & 1.67 \\
\hline $0.00005-0.000005$ & $3.2 \cdot 10^{-5}$ & 5.3 & 0.86 & 1.87 \\
\hline $0.00005-0.0000025$ & $4.2 \cdot 10^{-5}$ & 8.48 & 0.86 & 1.54 \\
\hline
\end{tabular}

In Table 6.2 errors in the $L^{2}\left(0, T ; L^{2}(\Omega)\right)$ and $L^{2}\left(0, T ; H^{1}(\Omega)\right)$ norms (resp. $e_{L^{2}}$ and $\left.e_{H^{1}}\right)$ are reported when using anisotropic meshes $(h 1-h 2$ denotes the mesh size in horizontal and vertical directions). Also, the effectivity indices $e i^{Z Z}$ and $e i^{A}$ corresponding to the Zienkiewicz-Zhu error estimator (3.21) and our simplified error indicator (5.3) are shown. Clearly, when the mesh is refined 

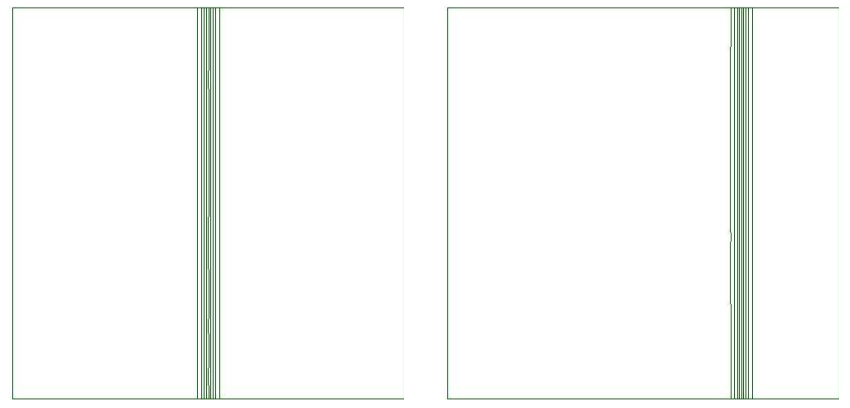

FIG. 6.1. Test case with exact solution: isolines (from 0.1 to 0.9 ) of $\phi$ and $c$ at time $t=0$ (left) and $t=0.5$ (right).

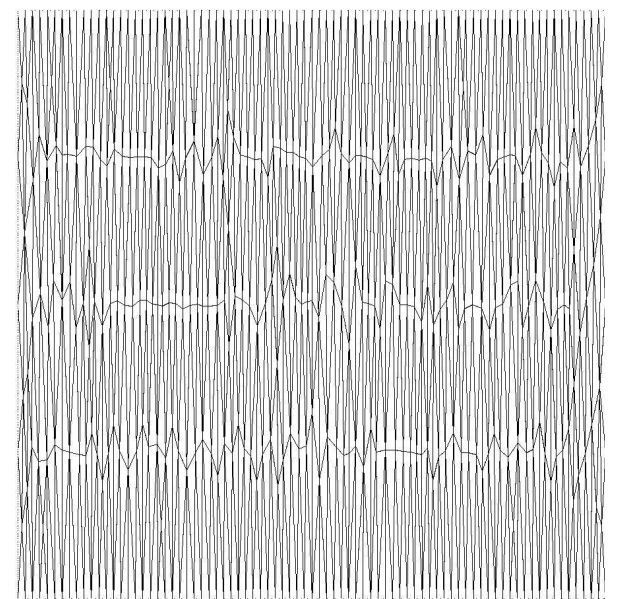

FIG. 6.2. Test case with exact solution: the mesh with size $h 1-h 2=0.000005-0.0001$.

in the horizontal direction, assumption $(3.4)$ holds with $s=1$ since the $L^{2}\left(0, T ; L^{2}(\Omega)\right)$ error converges at order two and the $L^{2}\left(0, T ; H^{1}(\Omega)\right)$ error at rate one. However, when the mesh is refined in the wrong (vertical) direction, then the error does not decrease and (3.4) does not hold.

\subsection{Computations with small anisotropy}

We now consider the following physical situation. At initial time, the computational domain is liquid, with homogeneous concentration 0.02. Then a circular solid seed of diameter $2.5 \cdot 10^{-6}$ and concentration 0.015 is placed at the center of $\Omega$. The physical parameters are now given in Table 6.3 and are taken from [15, Table 1, column B], except $c_{s}$ and $c_{l}$.

TABLE 6.3

Parameters used for the computations

\begin{tabular}{cccccccc}
\hline$\lambda$ & $m_{l}$ & $\Gamma$ & $c_{s}$ & $c_{l}$ & $D_{s}$ & $D_{l}$ & $\mu_{k}$ \\
\hline $0.5 \cdot 10^{-6}$ & -260 & $5 \cdot 10^{-7}$ & 0.015 & 0.0238 & $5 \cdot 10^{-10}$ & $5 \cdot 10^{-9}$ & 0.0015 \\
\hline
\end{tabular}



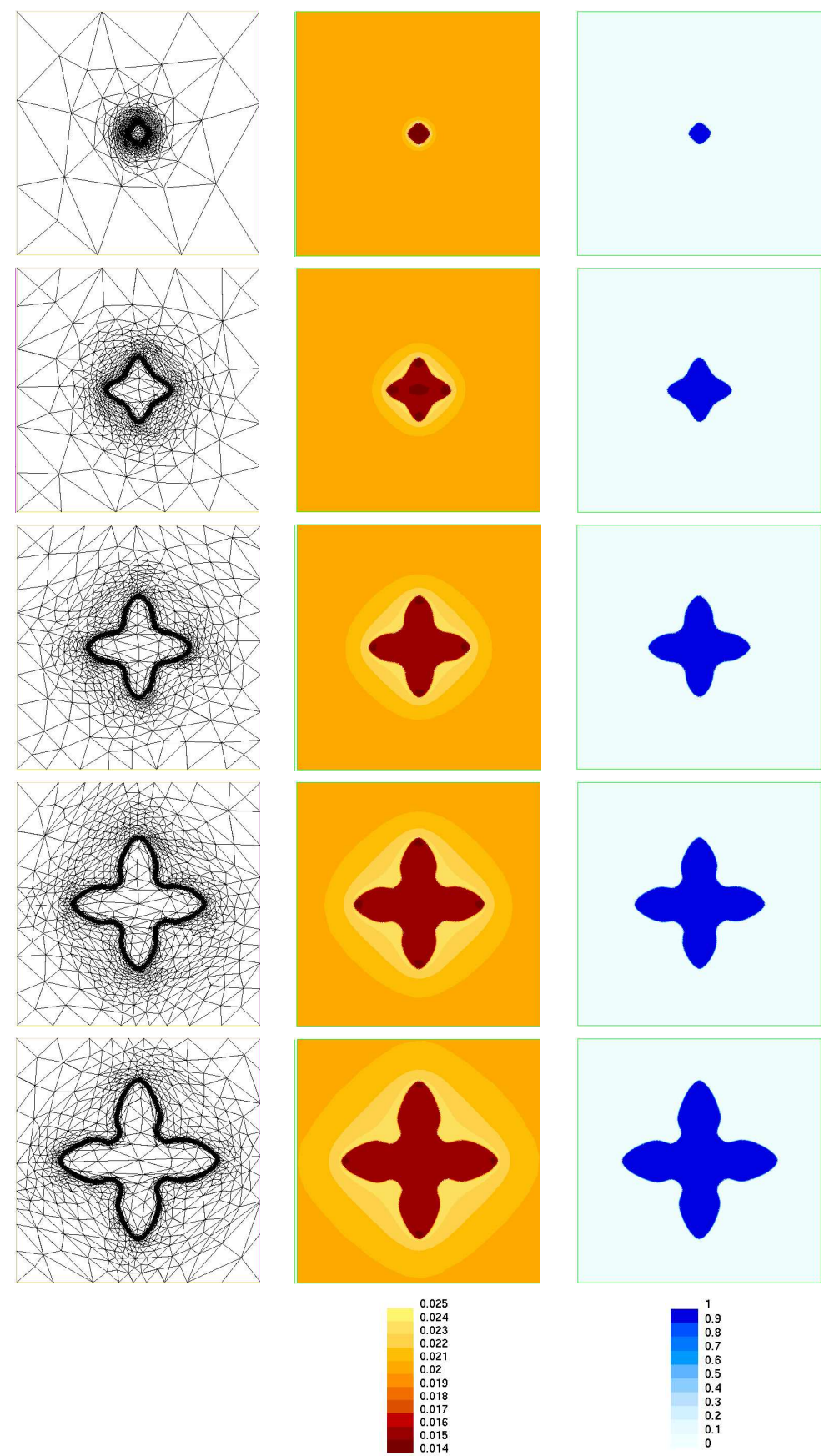

1.9
0.9
0.9
0.7
0.6
0.5
0.4
0.3
0.2
0.1
0

FIG. 6.3. Computations with small anisotropy, $\bar{a}=0.04$. Adapted meshes (left column), concentration isovalues (middle column) and phase isovalues (right column), from $t=0$ to $t=1 \mathrm{~s}$, with TOL $=0.0625$ (6.25\% estimated relative error). Row 1: $t=0.05 \mathrm{~s}, 6874$ vertices. Row $2: t=0.25 \mathrm{~s}, 10449$ vertices. Row $3: t=0.5 \mathrm{~s}, 17170$ vertices. Row $4: t=0.75 \mathrm{~s}$, 21795 vertices. Row 5: $t=1 \mathrm{~s}, 24441$ vertices. 


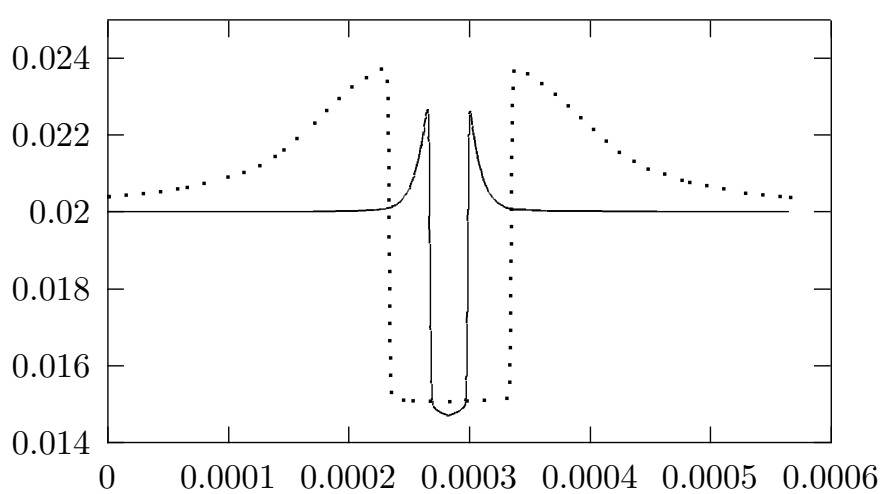

FIG. 6.4. Computations with small anisotropy, $\bar{a}=0.04$. Concentration profile along the diagonal of the computational domain at time 0.05 (solid line) and $1 \mathrm{~s}$ (dotted line).
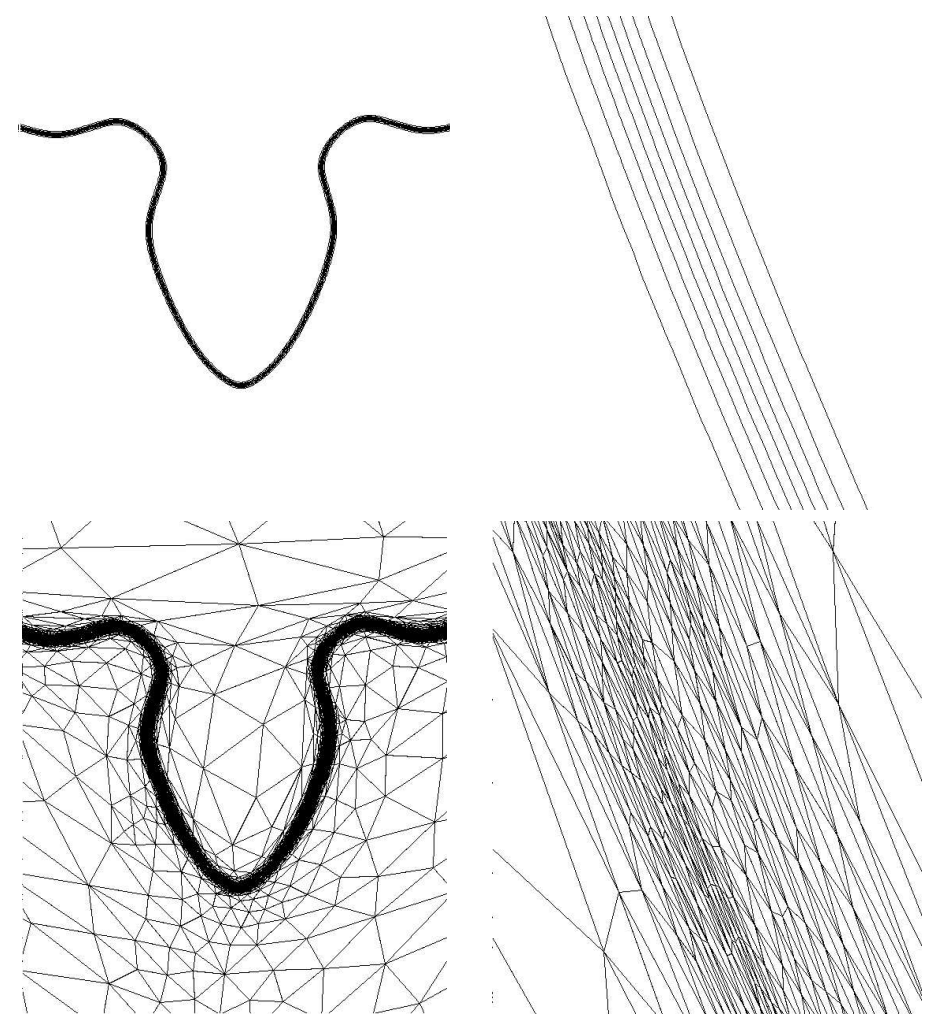

FIG. 6.5. Computations with small anisotropy, $\bar{a}=0.04$. Zooms of the results at final time. Top row: isolines of $\phi$ from 0.1 to 0.9 . Bottom row: mesh 

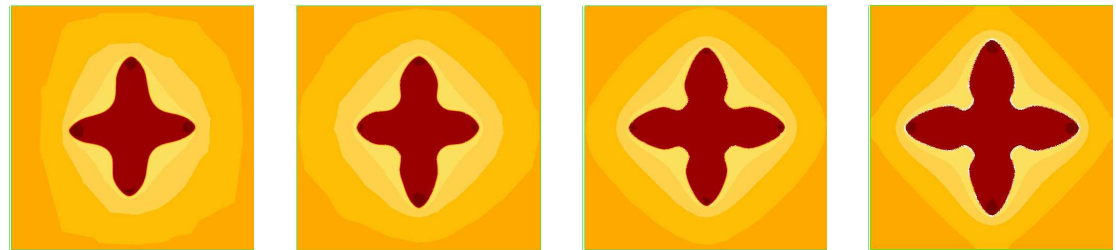

FIG. 6.6. Computations with small anisotropy, $\bar{a}=0.04$. Concentration isovalues at final time $t=1 \mathrm{~s}$ with various values of the tolerance TOL. From left to right: TOL $=0.25$, final adapted mesh 1987 vertices; TOL $=0.125,6073$ vertices; TOL $=0.0625,24441$ vertices; $\mathrm{TOL}=0.03125,101782$ vertices.

We first present computations in the case when the anisotropy parameter $\bar{a}$ is small. We set the number of dendrite arms $\kappa=4$ and choose $\bar{a}=0.04$ so that $\bar{a}<1 /\left(\kappa^{2}-1\right) \simeq 0.0667$. The time step is $\tau=5 \cdot 10^{-4}$ and the final time is $t=1$, making the total number of time steps 2000 .

In Fig. 6.3, the adapted meshes, concentration and phase fields corresponding to an adaptive computation with tolerance TOL $=0.0625(6.25 \%$ estimated relative error) are reported. The concentration profile along the diagonal of the computational domain is reported in Fig. 6.4 The concentration $c$ and phase $\phi$ appear to be smooth, but exhibit strong gradients across the solid-toliquid transition zone, therefore the mesh is strongly refined in the neighbourhood of the solid-liquid interface. Zooms of the results are shown in Fig. 6.5. The adaptive algorithm generates 400 meshes from initial to final time. The computation takes about 4 hours on a Pentium III 1.2 Ghz PC, with a required memory of less than $300 \mathrm{Mb}$. Convergence of the finite element solution with respect to the mesh size is reported in Fig. 6.6. The maximal anisotropy ratio of the mesh during a simulation was approximately 30 , without any a priori upper bound imposed by the adaptive method.

\subsection{Computations with large anisotropy}

We now choose the anisotropy parameter $\bar{a}>1 /\left(\kappa^{2}-1\right) \simeq 0.0667$, namely $\bar{a}=0.1$. In this case there are no known existence results for the system in $L^{2}\left(0, T ; H^{1}(\Omega)\right)$; in fact, only solutions in the Young measure sense are known to exist [8, 10]. However, due to the Lax-Milgram Lemma, the linearized problem (4.1) remains well-posed.
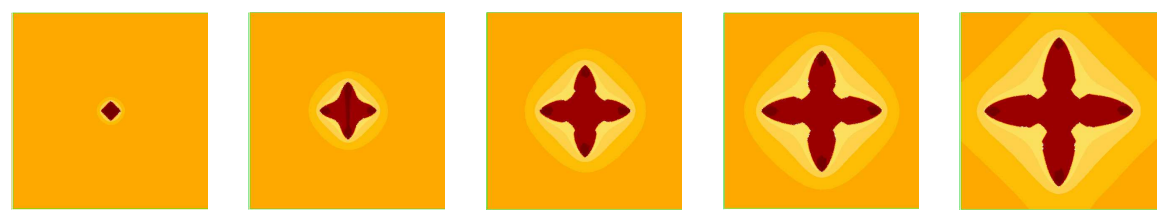

FIG. 6.7. Computations with large anisotropy, $\bar{a}=0.1$. Concentration isovalues from $t=0$ to $t=1 \mathrm{~s}$, with TOL $=0.0625$ $(6.25 \%$ estimated relative error). Column $1: t=0.05 \mathrm{~s}, 5138$ vertices. Column $2: t=0.25 \mathrm{~s}, 12053$ vertices. Column 3 : $t=0.5 \mathrm{~s}, 20580$ vertices. Column $4: t=0.75 \mathrm{~s}, 28605$ vertices. Column 5: $t=1 \mathrm{~s}, 29971$ vertices.

In Fig. 6.7 the concentration fields corresponding to an adaptive computation with tolerance TOL $=0.0625$ are reported. A zoom of the results at final time, Fig. 6.8, shows that the gradient is discontinuous in some regions, for instance close to the dendrite tip. We explain this phenomenon in the following manner. 

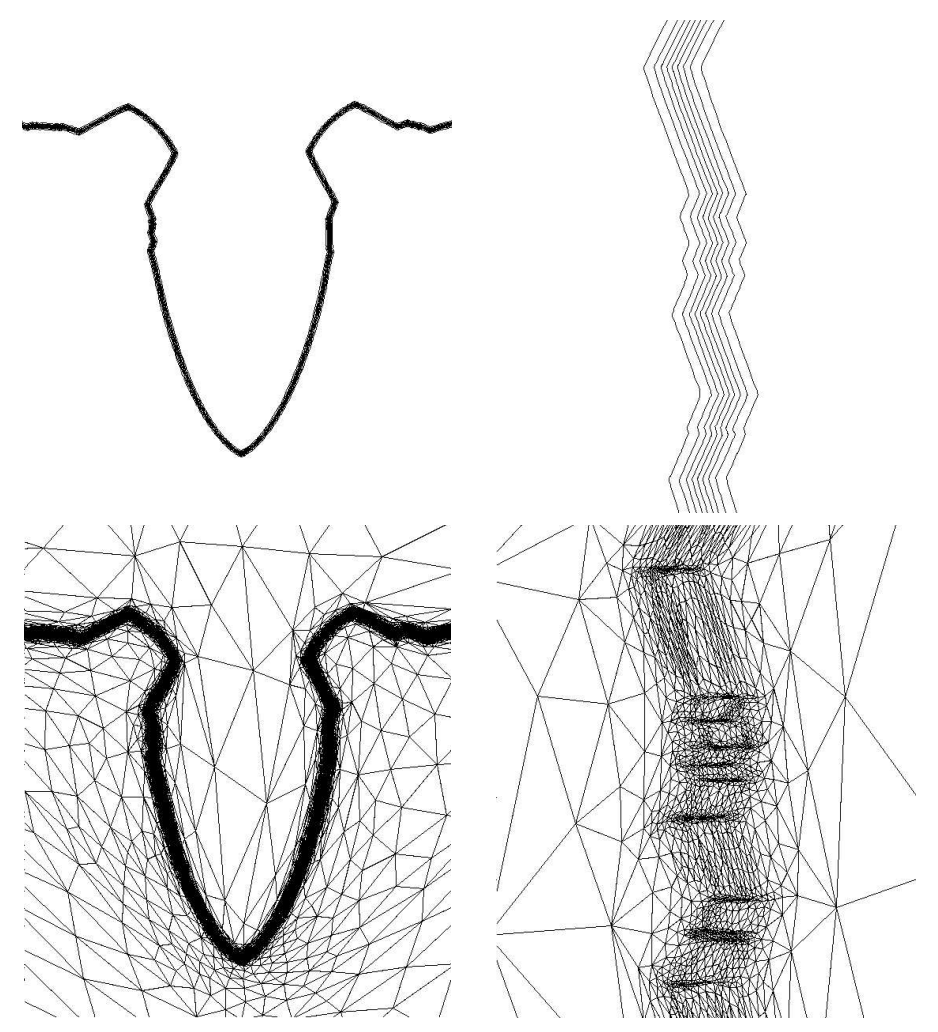

FIG. 6.8. Computations with large anisotropy, $\bar{a}=0.1$. Zooms of the results at final time. Top row: isolines of $\phi$ from 0.1 to 0.9. Bottom row: mesh.

Let us consider the functional

$$
\int_{\Omega} g(\nabla \phi) \mathrm{d} x,
$$

where $g(\xi)=a^{2}(\theta(\xi))|\xi|^{2} / 2$ and whose Fréchet derivative is $\operatorname{div}(A(\nabla \phi) \nabla \phi)$. In Fig. 6.9, we have plotted isolines of the anisotropic function $\xi \mapsto a^{2}(\theta(\xi))|\xi|^{2}$ with small and large anisotropy. As proved in [8], this function is convex if $\bar{a}<1 /\left(\kappa^{2}-1\right)$, this being the case when $\bar{a}=0.04$, but not when $\bar{a}=0.1$. However, when $\bar{a}>1 /\left(\kappa^{2}-1\right)$, the function $\xi \mapsto a^{2}(\theta(\xi))|\xi|^{2}$ is locally convex whenever $\xi$ satisfies

$$
|\cos \theta(\xi)| \leqslant\left|\cos \theta_{m}\right| \quad \text { and } \quad|\sin \theta(\xi)| \leqslant\left|\sin \theta_{m}\right|
$$

where $\theta_{m}$ solves

$$
a\left(\theta_{m}\right)+a^{\prime}\left(\theta_{m}\right) \frac{\cos \theta_{m}}{\sin \theta_{m}}=0 .
$$

When $\bar{a}=0.1$, this yields an angle $\theta_{m} \simeq 21.65$ degrees as reported in Fig. 6.9. In this zone the functional $g(\xi)$ coincides with its convex envelope $Q g(\xi)$ given by 

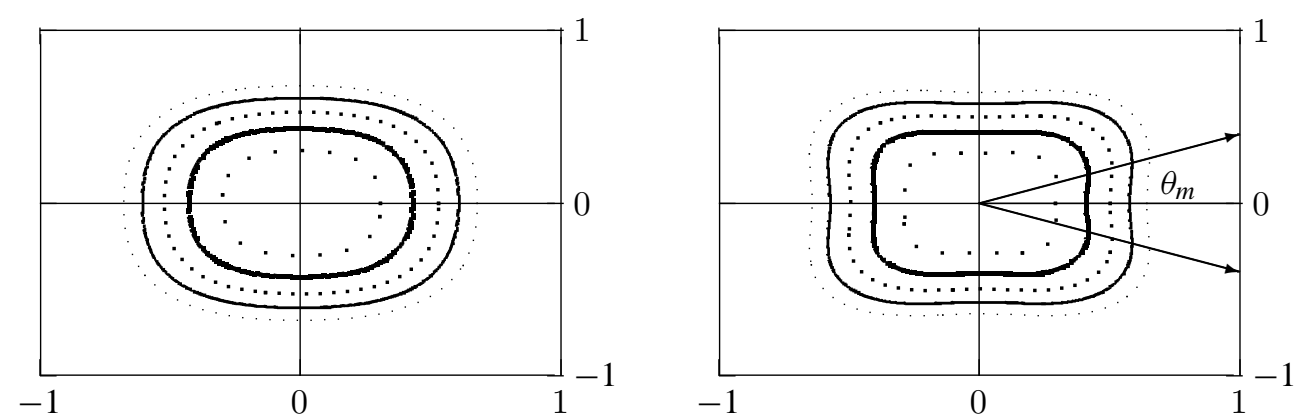

FIG. 6.9. Isolines $0.1,0.2,0.3,0.4,0.5$ of the function $\xi \mapsto a^{2}(\theta(\xi))|\xi|^{2}$ when $\bar{a}=0.04$ (left) and $\bar{a}=0.1$ (right).

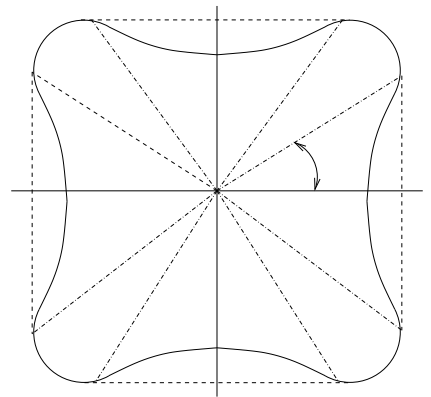

FIG. 6.10. Schematic representation of the anisotropic function $\xi \mapsto g(\xi)=a^{2}(\theta(\xi))|\xi|^{2}$ (solid line) and the corresponding convex envelope $\xi \mapsto Q g(\xi)$ (dotted line). The angle reported in the figure corresponds to $\theta_{m}$ such that $a\left(\theta_{m}\right)+$ $a^{\prime}\left(\theta_{m}\right) \frac{\cos \theta_{m}}{\sin \theta_{m}}=0$.

$$
Q g(\xi)=\left\{\begin{array}{cl}
\frac{a^{2}(\theta(\xi))|\xi|^{2}}{2} & \text { if }|\cos \theta(\xi)| \leqslant\left|\cos \theta_{m}\right| \text { and }|\sin \theta(\xi)| \leqslant\left|\sin \theta_{m}\right|, \\
\frac{a^{2}(\theta(\xi)) \xi_{1}^{2}}{2 \cos ^{2} \theta_{m}} & \text { if }|\cos \theta(\xi)|>\left|\cos \theta_{m}\right|, \\
\frac{a^{2}(\theta(\xi)) \xi_{2}^{2}}{2 \cos ^{2} \theta_{m}} & \text { if }|\sin \theta(\xi)|>\left|\sin \theta_{m}\right| ;
\end{array}\right.
$$

see Fig. 6.10 for a schematic representation of $Q g(\cdot)$. Turning once again to Fig. 6.8 we observe that the gradient jump corresponds to an angle approximately $2 \theta_{m}$ in the regions where the concentration gradient is discontinuous and that the oscillations do not seem to take place on the scale of the mesh but are distributed with different distances. Thus, as expected, $\nabla \phi$ avoids the high energy directions where

$$
|\cos \theta(\nabla \phi)|>\left|\cos \theta_{m}\right| \quad \text { or } \quad|\sin \theta(\nabla \phi)|>\left|\sin \theta_{m}\right|
$$

that is to say, the region where the function $\xi \mapsto a^{2}(\theta(\xi))^{2}|\xi|^{2}$ is nonconvex. It is however very difficult to draw any conclusions whether or not the dendritic branches exhibit microstructure. In order to examine these effects closer we propose to make computations using the convexified problem with $\operatorname{div}(A(\nabla \phi) \nabla \phi)$ replaced by the Fréchet derivative of 


$$
\int_{\Omega} Q g(\nabla \phi) \mathrm{d} x+\sum_{K \in \mathcal{T}_{h}} \lambda_{2, K}^{2} \int_{K}|\nabla \phi|^{2} \mathrm{~d} x
$$

where $\lambda_{2, K}$ is defined as in Section 2. The last term in the above convexified functional corresponds to a mesh dependent artificial viscosity added to counter the loss of coercivity in the zones where $g(\xi)>Q g(\xi)$.

In Fig. 6.11 we have reported results corresponding to the convexified functional with $\bar{a}=0.1$ and TOL $=0.0625$. Comparing with Fig. 6.8, we observe that the general form of the dendrite branches remains the same, with sharp dendrite tips and sharp corners at the base of the branches. However the regions on the sides of the branches where $\phi_{h}$ is nonsmooth have disappeared. Here the gradient of the solution of the convexified problem $\phi_{h}^{c}$ points in the high energy direction, so that $\int_{\Omega}\left(g\left(\nabla \phi_{h}^{c}\right)-Q g\left(\nabla \phi_{h}^{c}\right)\right) \mathrm{d} x>0$. Furthermore this last quantity does not decrease with decreasing tolerance. This numerical result suggests that after a certain time $T^{*}$ there is no $H^{1}(\Omega)$ solution to problem $11.1-(1.5)$ when $\bar{a}>1 /\left(\kappa^{2}-1\right)$, but only Young measure solutions, with microstructure appearing on the sides of the dendrite branches. In other words, the approximating sequence converges to a solution of the problem only in the sense of Young measures, where an admissible Young measure (a priori nonunique) can be derived from the corresponding convexification 6.1.
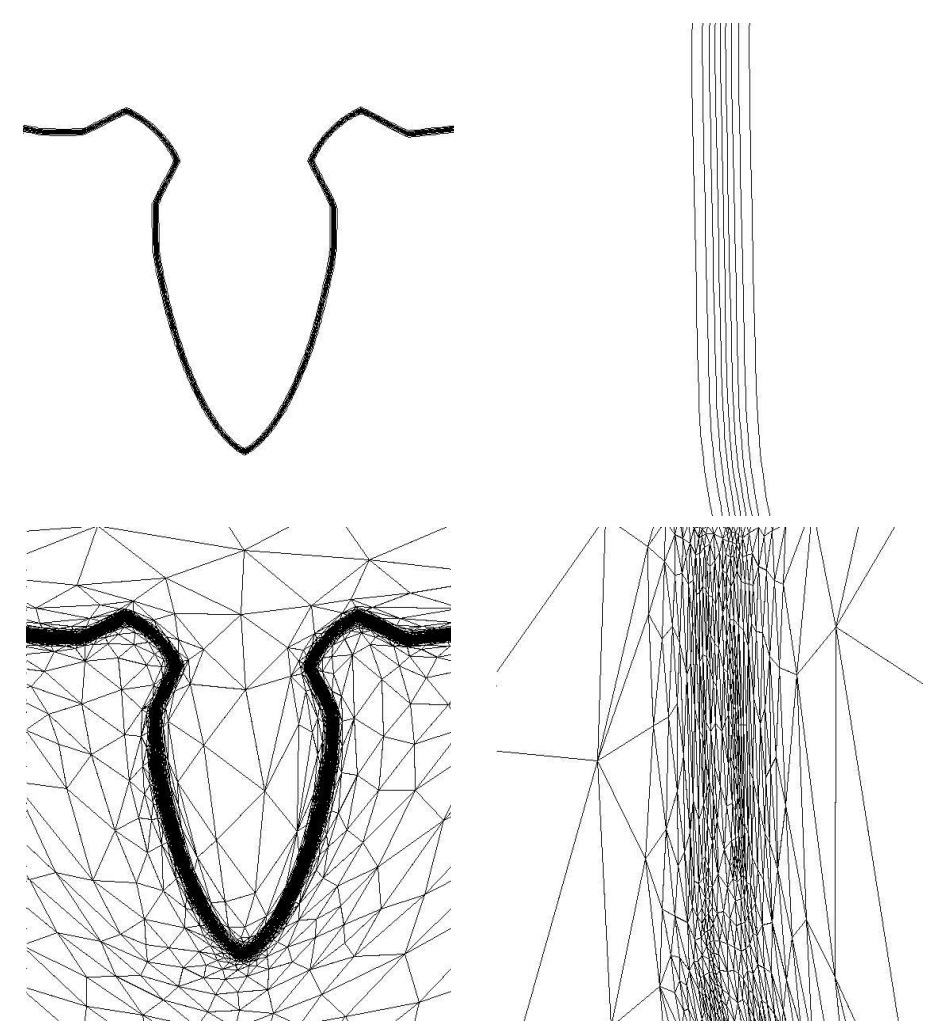

FIG. 6.11. Computations with large anisotropy, $\bar{a}=0.1$. Convexified functional. Zooms of the results at final time. Top row: isolines of $\phi$ from 0.1 to 0.9 . Bottom row: mesh. 


\section{REFERENCES}

1. Ainsworth, M. \& Oden, J. T. A posteriori error estimation in finite element analysis. Comput. Methods Appl. Mech. Engrg. 142 (1997), 1-88. Zbl 0895.76040 MR 98e:65092

2. Ainsworth, M. \& Oden, J. T. A unified approach to a posteriori error estimation using finite element residual methods. Numer. Math. 65 (1993), 23-50. Zbl 0797.65080 MR 95a:65185

3. Ainsworth, M., Zhu, J. Z., Craig, A. W., \& Zienkiewicz, O. C. Analysis of the Zienkiewicz-Zhu a posteriori error estimator in the finite element method. Internat. J. Numer. Methods Engrg. 28 (1989), 2161-2174. Zbl 0716.73082 MR 90j:73053

4. Babuška, I., Strouboulis, T., \& Upadhyay, C. S. A model study of the quality of a posteriori error estimators for linear elliptic problems. Error estimation in the interior of patchwise uniform grids of triangles. Comput. Methods Appl. Mech. Engrg. 114 (1994), 307-378. MR 95d:65093

5. BorouchaKi, H. \& LAUG, P. The bl2d mesh generator: Beginner's guide, user's and programmer's manual. Technical Report RT-0194, Institut National de Recherche en Informatique et Automatique (INRIA), Rocquencourt, Le Chesnay, 1996.

6. Brezis, H. Analyse fonctionnelle, Théorie et applications. Masson, 1983. Zbl 0511.46001 MR 85a:46001

7. Burman, E., Kessler, D., \& RapPAZ, J. Convergence of the finite element method for an anisotropic phase-field model. Technical report, Département de Mathématiques, Ecole Polytechnique Fédérale de Lausanne, 2002.

8. Burman, E. \& RAppaz, J. Existence of solutions to an anisotropic phase-field model. Math. Methods Appl. Sci., to appear.

9. CARSTEnsen, C. \& Verfürth, R. Edge residuals dominate a posteriori error estimates for low order finite element methods. SIAM J. Numer. Anal. 36 (1999), 1571-1587. Zbl 0938.65124 MR 2000g:65115

10. Demoulini, S. Young measure solutions for a nonlinear parabolic equation of forward-backward type. SIAM J. Math. Anal. 27 (1996), 376-403. Zbl 0851.35066 MR 97a:35096

11. Elliott, C. M. Approximation of curvature dependent interface motion. The State of the Art in Numerical Analysis (York, 1996), Oxford Univ. Press, New York, 1997, 407-440. Zbl 0881.65131 MR 99c:35120

12. Elliott, C. M. \& Stuart, A. M. The global dynamics of discrete semilinear parabolic equations. SIAM J. Numer. Anal. 30 (1993), 1622-1663. Zbl 0792.65066 MR 94j:65127

13. Formaggia, L. \& Perotto, S. Anisotropic error estimates for elliptic problems. Numer. Math., to appear.

14. Formaggia, L. \& Perotto, S. New anisotropic a priori error estimates. Numer. Math. 89 (2001), 641-667. Zbl 0990.65125 MR 2002j:65110

15. JACOT, A. \& RAPPAZ, M. A pseudo front tracking technique for the modelling of solidification microstrucures in multicomponent alloys. Technical report, Département des Matériaux, Ecole Polytechnique Fédérale de Lausanne.

16. Kessler, D. \& SCHEID, J.-F. A priori error estimates for a phase-field model for the solidification process of a binary alloy. IMA J. Numer. Anal. 22 (2002), 281-305. Zbl pre01786767| MR 1897410

17. Kruger, O., PiCAsso, M., \& SCheid, J.-F. A posteriori error estimates and adaptive finite elements for a nonlinear parabolic problem arising from solidification. Comput. Methods Appl. Mech. Engrg. 192 (2003), 535-558.

18. PicAsso, M. Numerical study of the effectivity index for an anisotropic error indicator based on Zienkiewicz-Zhu error estimator. Comm. Numer. Methods Engrg. 19 (2003), 13-23. MR 1952014

19. PicAsso, M. An anisotropic error indicator based on zienkiewicz-zhu error estimator: application to elliptic and parabolic problems. SIAM J. Sci. Comput. 24 (2003), 1328-1355. 
20. RAPPAZ, J. \& SCHEID, J.-F. Existence of solutions to a phase-field model for the isothermal solidification process of a binary alloy. Math. Methods Appl. Sci. 23 (2000), 491-513. Zbl 0964.35026 MR 2001i:35159

21. Rodríguez, R. Some remarks on Zienkiewicz-Zhu estimator. Numer. Methods Partial Differential Equations 10 (1994), 625-635. Zbl 0806.73069 MR 95e:65103

22. Tiaden, J., Nestler, B., Diepers, H. J., \& Steinbach, I. The multiphase-field model with an integrated concept for modelling solute diffusion. Phys. D 115 (1998), 73-86. Zbl 0956.74038

23. Warren, J. A. \& Boettinger, W. J. Prediction of dendritic growth and microsegregation patterns in a binary alloy using the phase-field model. Acta Metall. Mater. 43 (1995), 689-703.

24. ZiEnkiEwiCZ, O. C. \& ZHU, J. Z. A simple error estimator and adaptive procedure for practical engineering analysis. Internat. J. Numer. Methods Engrg. 24 (1987), 337-357. Zbl 0602.73063 MR 87m:73055

25. ZiEnkiewiCZ, O. C. \& ZHU, J. Z. The superconvergent patch recovery and a posteriori error estimates. I. The recovery technique. Internat. J. Numer. Methods Engrg. 33 (1992), 1331-1364. Zbl 0769.73084 MR 93c:73098 